\title{
A Phase I Study of Low-Pressure Hyperbaric Oxygen Therapy for Blast-Induced Post-Concussion Syndrome and Post-Traumatic Stress Disorder
}

\author{
Paul G. Harch, Susan R. Andrews, ${ }^{2}$ Edward F. Fogarty, ${ }^{3}$ Daniel Amen, ${ }^{4}$ John C. Pezzullo, ${ }^{5}$ \\ Juliette Lucarini, ${ }^{6}$ Claire Aubrey, ${ }^{6}$ Derek V. Taylor, ${ }^{4}$ Paul K. Staab,, and Keith W. Van Meter ${ }^{1}$
}

\begin{abstract}
This is a preliminary report on the safety and efficacy of 1.5 ATA hyperbaric oxygen therapy (HBOT) in military subjects with chronic blast-induced mild to moderate traumatic brain injury (TBI)/post-concussion syndrome (PCS) and post-traumatic stress disorder (PTSD). Sixteen military subjects received 401.5 ATA/60 min HBOT sessions in 30 days. Symptoms, physical and neurological exams, SPECT brain imaging, and neuropsychological and psychological testing were completed before and within 1 week after treatment. Subjects experienced reversible middle ear barotrauma (5), transient deterioration in symptoms (4), and reversible bronchospasm (1); one subject withdrew. Post-treatment testing demonstrated significant improvement in: symptoms, neurological exam, full-scale IQ $(+14.8$ points; $p<0.001)$, WMS IV Delayed Memory $(p=0.026)$, WMS-IV Working Memory $(p=0.003)$, Stroop Test $(p<0.001)$, TOVA Impulsivity $(p=0.041)$, TOVA Variability $(p=0.045)$, Grooved Pegboard $(p=0.028)$, PCS symptoms (Rivermead PCSQ: $p=0.0002)$, PTSD symptoms (PCL-M: $p<0.001$ ), depression (PHQ-9: $p<0.001$ ), anxiety (GAD-7: $p=0.007)$, quality of life (MPQoL: $p=0.003$ ), and self-report of percent of normal $(p<0.001)$, SPECT coefficient of variation in all white matter and some gray matter ROIs after the first HBOT, and in half of white matter ROIs after 40 HBOT sessions, and SPECT statistical parametric mapping analysis (diffuse improvements in regional cerebral blood flow after 1 and 40 HBOT sessions). Forty 1.5 ATA HBOT sessions in 1 month was safe in a military cohort with chronic blast-induced PCS and PTSD. Significant improvements occurred in symptoms, abnormal physical exam findings, cognitive testing, and quality-of-life measurements, with concomitant significant improvements in SPECT.
\end{abstract}

Key words: hyperbaric oxygen therapy; post-concussion syndrome; post-traumatic stress disorder; single photon emission computed tomography; traumatic brain injury

\section{Introduction}

B LAST-INDUCED TRAUMATIC BRAIN INJURY (TBI) and posttraumatic stress disorder (PTSD) are diagnoses of particular concern in the United States because of the volume of affected servicemen and women from the conflicts in Iraq and Afghanistan. A Rand report (Tanielian and Jaycox, 2008) estimates that $300,000(18.3 \%)$ of 1.64 million military service members who have deployed to these war zones have PTSD or major depression, and 320,000 (19.5\%) have experienced a TBI. Overall, approximately 546,000 have one of the three diagnoses, and 82,000 have symptoms of all three (symptoms of TBI refer to the post-concussion syndrome [PCS]). The frequency of the combined diagnoses in veterans of mild TBI and PTSD has recently been estimated to be between 5 and $7 \%$ (Carlson, 2010). With a probable diagnosis of mild TBI the combined diagnosis incidence rises to 33-39\% (Carlson, 2010). A Walter Reed Army Institute of Research post-deployment survey of 4618 soldiers reported that $15.2 \%$ of the injured had a history of loss of consciousness or altered mental status (Hoge et al., 2008). That study also found that $43.9 \%$ of those with a history of loss of consciousness and

\footnotetext{
${ }^{1}$ Hyperbaric Medicine Department, Department of Medicine, Section of Emergency and Hyperbaric Medicine, ${ }^{2}$ Department of Medicine and Psychiatry, School of Medicine, Louisiana State University Health Sciences Center, New Orleans, Louisiana.

${ }^{3}$ Department of Radiology, University of North Dakota School of Medicine and Health Sciences, Bismarck, North Dakota.

${ }^{4}$ University of California, Irvine, School of Medicine, Amen Clinics, Inc., Newport Beach, California.

${ }^{5}$ Department of Medicine, Georgetown University Medical Center, Washington, D.C.

${ }^{6}$ Administrative Office, New Orleans, Louisiana.
} 
$27.3 \%$ of those with a history of altered mental status met criteria for PTSD.

Evidence-based treatment for PTSD exists, but problems with access to and quality of treatment have been problematic in the military setting (Tanielian and Jaycox, 2008). Treatment of the symptomatic manifestations of mild TBI, the PCS, is limited. Treatment consists of off-label use of FDA black-box labeled psychoactive medications, counseling, and stimulative and adaptive strategies. There is no effective treatment for the combined diagnoses of PCS and PTSD. The purpose of this study is to explore the feasibility, safety, and treatment effects of hyperbaric oxygen therapy on PCS and PTSD.

Hyperbaric oxygen therapy (HBOT) is a medical treatment that uses greater than ambient pressure oxygen as a drug by fully enclosing a person or animal in a pressure vessel and then adjusting the dose of the drug to treat pathophysiologic processes of diseases (Harch and Neubauer, 1999). At 2.0-3.0 atmospheres absolute (ATA) HBOT is a reimbursed treatment for approximately 15 diagnoses (Centers, 2006; Gesell, 2009). HBOT has not been applied to PTSD to our knowledge, while the evidence for its effectiveness in PCS is scant. Since 1989 we and others have investigated the application of a lower-pressure protocol of HBOT, HBOT 1.5 ATA, to patients with a variety of chronic neurological disorders (Golden et al., 2002; Harch and Neubauer, 1999,2004a,2009b,2009c; Harch et al., 1994, 1996a; Neubauer et al., 1994), based on initial studies done by Neubauer (Neubauer et al., 1990) in chronic stroke. Few of the chronic TBI patients had PCS from mild TBI, blast-induced PCS, or blast-induced PCS with PTSD. We published the first application of HBOT 1.5 ATA to chronic blast-induced PCS with PTSD in 2009 (Harch and Fogarty, 2009a).

Oxygen toxicity (Clark, 1993; U.S. Navy, 2008) is a concern in any HBOT study. The most severe manifestation is seizure. A study of HBOT on sub-acute moderate to severe TBI at 2.0 ATA (Lin et al., 2008) reported a 9\% seizure rate. At doses less than 2.0 ATA, side effects and toxicity in chronic brain injured patients have been noted only with prolonged courses of HBOT (i.e., 70-500 treatments [Harch, 2002]).

We report the safe application of a 29-day treatment course of 1.5 ATA HBOT to 16 U.S. servicemen with mild to moderate blast-induced PCS, or PCS with PTSD, and note a biphasic response, with transient worsening of symptoms in 4 of the 16 subjects, followed by improvement as treatment continued. These veterans experienced symptomatic, physical, cognitive, affective, and brain blood flow improvements.

\section{Methods}

\section{Study design and protocol}

The design is a pilot proof-of-concept study with pre- and post-testing and no control group. Subjects completed a history and physical exam by the P.I., clinical interview by the neuropsychologist, psychometric testing, symptom and quality-of-life questionnaires, baseline single photon emission computed tomography (SPECT), first HBOT the following day, and repeat SPECT $3 \mathrm{~h}$ after the first HBOT. Subjects commenced twice/day, 5 day/week 1.5 ATA/60 min total dive time HBOT until 40 HBOT sessions were completed. Within 5 days of final HBOT subjects underwent repeat focused history, physical exam, psychometric testing, questionnaires, and SPECT.

\section{Inclusion criteria}

Subjects had to be 18-65 years old, with one or more mild to moderate TBIs characterized by loss of consciousness due to blast injury that was a minimum of 1 year old and occurred after $9 / 11 / 01$. They had to have a prior diagnosis of chronic TBI/PCS or TBI/PCS/PTSD by military or civilian specialists, with an absence of acute cardiac arrest or hemorrhagic shock at the time of TBI, Disability Rating Scale score (Rappaport et al., 1982) of 0-3, negative urine toxicology screen for drugs of abuse, negative pregnancy test in females, otherwise good health, and less than $90 \%$ on the Percent Back to Normal Rating Scale (PBNRS; Powell et al., 2001).

\section{Exclusion criteria}

Subjects were screened out of the study with pulmonary disease that precludes HBOT (e.g., bronchospasm unresponsive to medication or bullous emphysema), unstable medical conditions that are contraindicated in HBOT (e.g., severe congestive heart failure or heart failure requiring hospital emergency evaluation or admission in the previous year), severe confinement anxiety (e.g., patients who require anesthesia or conscious sedation for MRI), participation in another experimental trial with active interventions, high probability of inability to complete the experimental protocol (e.g., terminal condition), previous HBOT, history of hospitalization for past TBI, stroke, non-febrile seizures, or any seizure history other than seizure at the time of TBI, past or current history of mental retardation (baseline full-scale intelligence quotient [FSIQ] score $\leq 70$ ), alcohol or drug abuse (Michigan Alcohol Screening Test [MAST] or Drug Abuse Screening Test score [DAST] >3), or pre- or post-TBI history of systemic illness with impact on the CNS (per P.I. decision).

\section{Symptom and physical exam scoring}

Subjects constructed a prioritized symptom list and answered neurological and constitutional symptom questions from the P.I.'s standard questionnaire (see Appendix). Abnormal components of the physical exam were videotaped and then replayed before the final exam after HBOT for comparison. After the 40th HBOT session, subjects judged all symptoms as "better," "worse," or "same," and the P.I. did the same for the physical exam abnormalities. Exam items inadvertently omitted on retesting were scored as unchanged. Six months following the last HBOT session subjects were queried by phone about the status of their prioritized symptom list. Each subject was asked to rate each symptom as better, worse, or the same compared to the status of that symptom before HBOT.

\section{Psychometric testing}

Table 1 lists neuropsychological and psychological, quality of life, screening and diagnostic tests, and the schedule of administration. The choice of tests was guided by past experience with pre- and post-testing for HBOT effects in chronic TBI. IQ testing was included instead of more easily measured variables like reaction time, because of a concern that the measures reflect social relevance and the reported deficits from injury, such as frontal lobe (attention, executive function, motor speed, decision speed, and working memory), general intellectual ability, memory, PCS symptoms, quality of life, 
and affective symptoms (anxiety or depression). Practice and test/retest effects were minimized by choice of tests, or where possible using alternate tests (e.g., Wechsler Adult Intelligence Scale-IV [WAIS-IV] on pre-test and Wechsler Abbreviated Scale of Intelligence [WASI] post-test). All tests were outcome tests except the Wechsler Test of Adult Reading (WTAR), Green, MAST, DAST, and Combat Experience Scale (CES). The original screening PBNRS is defined in Table 1. It was expanded at psychometric test sessions to include cognitive, emotional, and physical domains, and each subject was asked to rate his or her current percent of premorbid normal function in each domain. Prior diagnoses of TBI/PCS and PTSD were confirmed or refuted by using clinical interviews, symptom lists, the Rivermead Post Concussion Symptoms Questionnaire (PCS: $\geq 3$ on at least 3 questions [Sterr et al., 2006]), the PTSD Checklist-Military (total $\geq 50$; Andrykowski et al., 1998; Tanielian and Jaycox, 2008), and Diagnostic and Statistical Manual-IV (DSM-IV) criteria for the diagnoses.

\section{SPECT brain blood flow imaging}

Subjects underwent SPECT brain blood flow imaging performed by a single technologist on a Picker Prism 3000 XP Triple-Head gamma camera system before, within $4 \mathrm{~h}$ after the first HBOT session, and within $48 \mathrm{~h}$ after the 40th HBOT session. Subjects were placed on a gurney in the supine position, with the head of the bed elevated $30^{\circ}$ degrees, in a designated quiet low-light area of the nuclear medicine department. Heparin lock IV catheter was placed and after at least $15 \mathrm{~min}$ of no speech or movement $\sim 25 \mathrm{mCi}$ of ${ }^{99 \mathrm{~m}} \mathrm{Tech}-$ netium ethyl cysteinate dimer (ECD) was injected and followed with a $10 \mathrm{cc}$ normal saline flush. The patient remained quiet and motionless for another $55 \mathrm{~min}$, and then was placed supine on the scanning couch. The head was secured with tape to the head cradle and the subject was aligned with an overhead laser. Acquisition entailed a $360^{\circ}$ rotation with 40 stops, $20 \mathrm{sec} /$ stop, on a $128 \times 128$ matrix, using low-energy high-resolution fan beam collimators. Cine was viewed for gross motion artifacts and the study was immediately repeated if the image was motion degraded.

Processing was performed by a single off-site experienced nuclear technologist. Mild motion artifact was corrected with Picker motion attenuation software. Raw data were processed by transverse reconstruction using $360^{\circ}$ filtered back projection and a ramp filter, followed by a LoPass filter, order 2.2. Cut-off was taken at the intersection of the best fit LoPass filter and noise on the power spectrum graph. Per file attenuation correction and best fit ellipse were applied. Images were oblique reformatted with slice thickness at $4 \mathrm{~mm}$ (2 pixels), aligned, and off-center zoom was applied $\left(20 \mathrm{~cm}^{2}\right.$ area). Images were presented in all 3 orthogonal planes.

\section{SPECT texture analysis}

Transverse processed images were analyzed by author E.F.F. (unblinded to study and scan sequence) to capture the pre-/post-HBOT SPECT pattern change from heterogeneity to homogeneity (Fig. 1) that we have observed in previous HBOT-treated patients (Harch et al., 1996a, 2009a; Harch and Neubauer, 2009c). Osirix ${ }^{\mathrm{TM}}$ DICOM software was used to perform a first-order texture analysis of count histograms (Dougherty, 1996). In previous HBOT-treated blast cases the pattern shift (apparent normalization) corresponded to a relative reduction in high flow areas, and a relative increase in low flow areas (insets in Fig. 1), or narrowing of the count histogram that was registered as a reduction in standard deviation of counts / pixel (SD), and coefficient of variation (CV) (see below).

Images were oriented and aligned by visual inspection. A single transverse slice was taken above the level of the deep gray matter in the centrum semiovale of each patient's three SPECT brain scans. A circular region of interest (ROI) was chosen of sufficient size, $0.781 \mathrm{~cm}^{2}$, to fit within the cortical boundary of the baseline (first) scan. Five cortical and two

Table 1. List of Psychometric Measures, When Administered, and Domain Measured

\begin{tabular}{|c|c|c|c|}
\hline & Pre & Post & Domain measured \\
\hline Combat Experience Scale (Keane et al., 1989) & $X$ & - & Combat experience \\
\hline Green Word Memory Test (Lesak et al., 2004) & $X$ & - & Effort \\
\hline Wechsler Test of Adult Reading (Wechsler, 2001) & $X$ & - & Estimate premorbid IQ \\
\hline Michigan Alcohol Screening Test (MAST Revised, 2009) & $X$ & $X$ & Alcohol use \\
\hline Drug Abuse Screening Test (Gavin et al., 1989) & $X$ & $X$ & Drug use \\
\hline $\begin{array}{l}\text { Percent Back To Normal Rating (Powell et al., 2001). Current } \\
\text { percent of normal premorbid level of function }\end{array}$ & $X$ & $x$ & Rating recovery \\
\hline Rivermead Post-Concussion Symptom Questionnaire (King et al., 1995) & $X$ & $X$ & PCS \\
\hline PTSD Checklist-Military (PTSD, 2009) & $X$ & $X$ & Rating PTSD \\
\hline Wechsler Adult Intelligence Scale-IV (WAIS-IV, 2009) & $X$ & & $\mathrm{IQ}$ \\
\hline Wechsler Abbreviated Scale of Intelligence (WASI, 2009) & & $X$ & IQ \\
\hline Test of Variables of Attention (Greenberg, 1996) & $X$ & $X$ & Attention \\
\hline Stroop Test (Lesak et al., 2004) & $X$ & $X$ & Attention, Executive function \\
\hline Finger Tapping Test (Reitan and Wolfson, 1993) & $X$ & $X$ & Motor speed \\
\hline Grooved Pegboard (Reitan and Wolfson, 1993) & $X$ & $X$ & Motor coordination \\
\hline Wechsler Memory Scale-IV (WMS-IV, 2009) & $X$ & $X$ & Memory, executive function \\
\hline Rivermead Paragraph Memory (Wilson et al., 1985) & $X$ & $X$ & Memory \\
\hline Perceived Quality Of Life (Patrick et al., 1988) & $X$ & $X$ & Satisfaction \\
\hline Patient Health Questionnaire-9 (Kroenke et al., 2001) & $X$ & $X$ & Depression \\
\hline Generalized Anxiety Disorder-7 (Spitzer et al., 2006) & $X$ & $X$ & Anxiety \\
\hline
\end{tabular}

PTSD, post-traumatic stress disorder; PCS, post-concussion syndrome; IQ, intelligence quotient. 


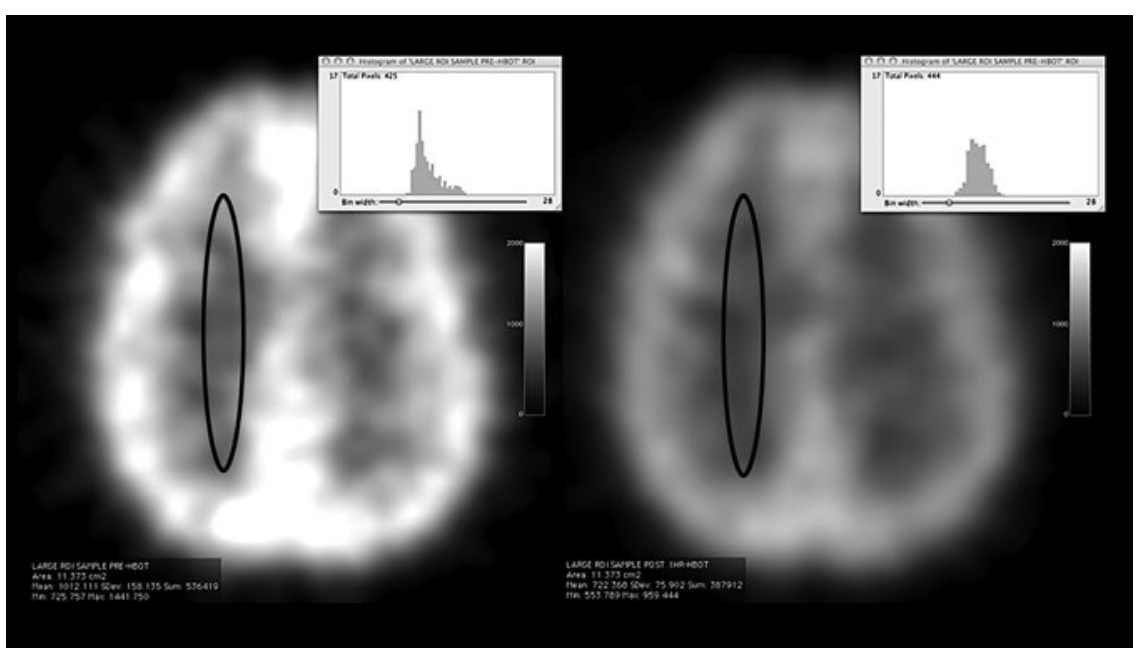

FIG. 1. Visual demonstration of single photon emission computed tomography (SPECT, gray scale) pattern change from heterogeneity (pre-HBOT, left) to homogeneity (post-one HBOT, right) in a sample transverse centrum semiovale slice. Inset histogram in each image shows counts in the white matter elliptical ROI (entire centrum white matter ROI was used for demonstration purposes only). Note the broader range of counts in the pre-HBOT scan than in the narrower concentration of counts post-1 HBOT. Visually, this is appreciated best in the cortical rim (HBOT, hyperbaric oxygen therapy; ROI, region of interest).

white matter ROIs were selected in each hemisphere. The cortical ROIs were placed along template ray-lines cast at $30^{\circ}$ angle intervals on either side of the anatomic center point in the image, assigning $0^{\circ}$ to $12: 00$ on a clock face of the transverse slice. The white matter ROIs were placed along the $60^{\circ}$ and $120^{\circ}$ ray-lines from the center point. Thus, the template ray-lines for the left and right hemispheres were at 1:00 and 11:00, respectively, for the $30^{\circ}$ ray, 2:00 and 10:00 for the $60^{\circ}$ ray, and so on. To aid best fit visualization for placement of the ROI on the second and third scans the pre-HBOT baseline image was individually fused in Osirix to the second and third scans. If the template ROIs landed across the cortical junction with white matter or across obvious focal metabolic lesion margins when first placed by whole scan best-fit fusion, they were adjusted along the ray-line to sample appropriate scanto-scan concordant tissues.

For each ROI mean number of counts/pixel (MCP), SD and $\mathrm{CV}$ (standard deviation as a percent of mean) of counts/pixel were measured for all three scans for each patient. Group averages for each ROI of mean counts/pixel, SD of counts/ pixel, and CV were taken for each scan's ROIs and the differences were compared from baseline to post- 1 and post-40HBOT scans. Statistical analysis was performed as described below. A decrease in CV was the primary SPECT outcome.

\section{SPECT statistical parametric mapping analysis}

Differences in ECD uptake were analyzed using SPM8 software (Wellcome Department of Cognitive Neurology, London, U.K.) implemented on the Matlab platform (MathWorks Inc., Sherborn, MA) by authors D.A. and D.V.T. Author D.V.T. performed all analyses and was asked to compare scan A to scan C, analyze for change, significance of change, and then direction of change, starting with a $p$ value for each voxel of $<0.01$. He was blinded to all details of the scans, including context (clinical study), patients/subjects, normal versus injury, treatment or not, one versus multiple groups, and location, and expectation of change or direction of change in the scans. D.V.T. was then asked to perform a similar comparison of scan B to A and C.

The images were spatially normalized using a 12-parameter affine transformation, followed by non-linear deformations (Ashburner and Friston, 1999) to minimize the residual sum of squares between each scan and a reference or template image conforming to the standard space defined by the Montreal Neurological Institute (MNI) template. The original image matrix obtained at $128 \times 128 \times 29$ with voxel sizes of $2.16 \times 2.16 \times 6.48 \mathrm{~mm}$ were transformed and resliced to a $79 \times 95 \times 68$ matrix with voxel sizes of $2 \times 2 \times 2 \mathrm{~mm}$, consistent with the MNI template. Images were smoothed using an 8-mm full-width half-maximum isotropic gaussian kernel. Withinsubject comparisons were performed by pair-wise $t$-test between the first and second scan, and between the first and third scan. Anatomical locations of the significant statistical parameter maps were identified by registering clusters using the Anatomical Automatic Labeling (AAL) atlas (Cyceron).

\section{Hyperbaric oxygen therapy}

Hyperbaric oxygen therapy was performed in monoplace hyperbaric chambers. Patients were compressed and decompressed at 1-2 psi (pounds per square inch) on 100\% oxygen, the rate depending on patient comfort and preference. Depth of pressurization was 1.5 ATA. Total dive time was $60 \mathrm{~min}$. Treatments were twice/day, 5 days/week, with a 3- to 4-h surface interval between treatments. Protocol goal was 40 HBOT sessions.

\section{Statistical analysis}

Values of psychometric tests were acquired pre- and post40 HBOT sessions, and SPECT parameters were acquired pre-, post- 1 HBOT, and post-40 HBOT sessions. For each SPECT ROI at each time point, mean, standard deviation, median, range (minimum and maximum), and 95\% confidence 
interval around the estimated mean were calculated for mean of counts/pixel, SD of counts/pixel, and CV of counts/pixel. Changes in psychometric and SPECT parameters between pairs of time points (pre-HBOT to post-1-HBOT, pre-HBOT to post-40-HBOTs, and post-1-HBOT to post-40-HBOTs) were similarly summarized, with the inclusion of a $p$ value indicating whether or not the mean change was significantly different from zero. The $p$ values were obtained by the paired Student's $t$-test if the changes were nearly normally distributed, or by the non-parametric Wilcoxon signed-ranks test if the changes were significantly non-normally distributed, by the Anderson-Darling test. One subject who withdrew before completion of treatment and post-treatment testing was included in the demographic data and safety/feasibility analysis, but excluded from the per protocol analysis (outcome testing).

\section{Results}

\section{Subjects}

Eight active duty and eight recently retired servicemen were self-referred or referred by their military commanders / physicians. Fourteen subjects had pre-study diagnoses of TBI/PCS with PTSD, and two subjects had TBI/PCS. Prestudy diagnostic evaluations and criteria were not available to the study authors. All subjects underwent brain MRI in the military prior to treatment. All subjects gave informed consent and enrolled in LSU IRB \#7051.

\section{Demographics of the sample}

Sample demographics are reported in Table 2. Sixteen subjects were enrolled. One subject withdrew from the study due to complications described below. Since he did not complete post-treatment testing he was included in the demographic data, but excluded from all data analyses. All subjects were male and averaged: 30 years old, 2.8 years post-TBI, loss of consciousness of $2 \mathrm{~min}$ (excluding 2 subjects with 4.5 and 9 h), 6 years of service, 2.7 blast TBIs, Rivermead Post Concussion Symptoms Questionnaire (RPCSQ) score 39, PTSD Checklist-Military (PCL-M) score 67, MAST 2.1, DAST .6, Disability Rating Score (DRS) 1.6, and 39 HBOTs in 29 days. Loss of consciousness (LOC) was estimated by each patient and the P.I. based on events at the time of injury and bystander reports to the patient. All 16 subjects satisfied the RPCSQ and DSM-IV criteria for PCS. Fifteen of sixteen subjects met the PCL-M threshold for PTSD $(\geq 50)$; the remaining subject scored 48. All 16 subjects met the DSM-IV criteria for PTSD.

\section{MRI results}

Results were obtained from patient recollection of results and medical records when available. Twelve of 16 subjects had normal MRIs of the brain. Two subjects were normal except for arachnoid cysts. Another had an abnormal MRI that was later repeated at the VA and reported as normal. A final subject had an abnormal MRI, but the abnormality was not recalled by the subject.

\section{Safety of the HBOT protocol}

Mild reversible middle ear barotrauma (MEBT) occurred in five subjects, four of these in the setting of upper respiratory
Table 2. Subject Demographic Characteristics

Safety population

Patient characteristic (all enrolled subjects)

\begin{tabular}{|c|c|}
\hline Number of subjects & 16 \\
\hline Sex & All male \\
\hline Average age (years, range) & $30(21-45)$ \\
\hline $\begin{array}{l}\text { TBI-to-HBOT interval } \\
\text { (years, range) }\end{array}$ & $2.8(1.25-4.75)$ \\
\hline Duration of LOC (min, range) & $\begin{array}{l}\text { Mean }=2.0 \text { for } 13 \text { subjects } \\
(1-10 \mathrm{~min}) ; \text { excluding } \\
2 \text { subjects ( } 4.5 \mathrm{~h} \text { and } 9 \mathrm{~h})\end{array}$ \\
\hline $\begin{array}{l}\text { Service at time of LOC } \\
\text { (years, range) }\end{array}$ & $6.0(1-17)$ \\
\hline $\begin{array}{l}\text { No. blast TBIs with LOC } \\
\text { or altered LOC }\end{array}$ & $2.7(1-7)$ \\
\hline RPCSQ score (scale: 0-64) & $39(27-47)$ \\
\hline PCL-M score (scale: 17-85) & $67(48-84)$ \\
\hline HBOTs/day & $\begin{array}{l}39 \text { HBOTs }(27-40) / \\
29 \text { days }(16-43)\end{array}$ \\
\hline MAST score (scale: $0-22$ ) & $2.1(0-3)$ \\
\hline DAST score (scale: $0-20$ ) & $0.6(0-3)$ \\
\hline Disability Rating (scale: 0-30) & $1.6(.5-3)$ \\
\hline PBNRS pre-study & $43 \%(5-72.5)$ \\
\hline Pre-TBI estimated IQ (average) & 104.9 \\
\hline Years of education (average) & 12.9 \\
\hline
\end{tabular}

Numerical variables are summarized as mean and range (minimum to maximum).

TBI, traumatic brain injury; IQ, intelligence quotient; PBNRS, Percent Back to Normal Rating Scale; DAST, Drug Abuse Screening Test; MAST, Michigan Alcohol Screening Test; HBOT, hyperbaric oxygen therapy; PCL-M, PTSD Checklist-Military; RPCSQ, Rivermead Post Concussion Symptoms Questionnaire; LOC, loss of consciousness.

infections at 8, 27, 27, and 30 HBOTs, requiring protocol breaks of 5 days, termination of protocol, 1 day, and 16 days, respectively. The fifth subject experienced no protocol break. All were treated with systemic decongestants with or without topical decongestants. Four of the five resumed treatment and successfully finished the protocol. The fifth subject experienced a series of problems that included a delay to scanning and treatment secondary to a scanner malfunction, followed by shortness of breath, beginning with the first HBOT, that was incident to each HBOT, and increased during his time in the chamber. Pre-/post-HBOT peak flow reductions were measured, he was medicated to symptom relief with albuterol pre-each HBOT, and showed a reduction in bronchospasm and shortness of breath with subsequent HBOTs. His bronchospasm was felt to be due to the low-humidity oxygen environment of the monoplace chamber. This subject subsequently experienced an upper respiratory infection (URI), MEBT, and bullous myringitis at 27 HBOTs. Because of the delay to testing caused by the scanner repair, the subject's schedule could not accommodate a protocol break to resolve the URI/MEBT and finish the protocol. He withdrew from the study and returned home.

Four of the sixteen subjects reported a transient deterioration in some of their symptoms: two with mood swings/ emotional lability at 20 and 10 HBOTs, one with worsened headaches at 19 HBOTs, and one with increased depression at 22-25 HBOTs. Treatment was continued and the symptoms resolved over the course of the next 4-6 HBOTs. There were 
no other untoward side effects. Specifically, we found no evidence of oxygen toxicity (Clark, 1993; U.S. Navy, 2008).

\section{Effectiveness of HBOT for chronic blast TBI/PCS and PTSD}

Effectiveness of HBOT was measured across multiple domains: symptoms, physical exam, psychometric testing, quality of life, and SPECT.

\section{Symptoms and physical exams}

Twelve of 15 subjects (80\%) reported improvement in a majority of their symptoms on their prioritized symptom list after HBOT. Eleven of 15 subjects (73\%) reported improvement in a majority of symptoms on the primary author's standard symptom questionnaire. Response to HBOT according to specific symptoms is recorded in Table 3, which combined symptoms from the prioritized list and the primary author's questionnaire. Headache, sleep disruption, shortterm memory loss, cognitive problems, decreased energy, self-characterized PTSD symptoms or nightmares (grouped as "PTSD symptoms," but not further queried or defined since PTSD symptomatology was quantified for all subjects in the PCL-M) short temper/irritability, mood swings, imbalance, photophobia, and depression, which were present in a majority of subjects, were improved in $44-93 \%$ of the subjects. Patients with decreased hearing, tinnitus, and arthralgias reported minimal change: 20,37 , and $0 \%$ improvement, respectively.

On physical exam all 15 subjects were found to have improved on a majority of their abnormal findings. Imbalance and incoordination were the most common abnormal physical exam findings (Table 4). Patients experienced improvement in $87-100 \%$ of these findings. In addition, $64 \%$ (7/11) of subjects who were on psychoactive or analgesic prescription medication before HBOT decreased or discontinued their medication use during HBOT; $11 \%$ of those on analgesic

Table 3. Symptom Changes (15 Subjects)

\begin{tabular}{lccc}
\hline Symptom & $\begin{array}{c}\text { Better } \\
(\%)\end{array}$ & $\begin{array}{c}\text { No change } \\
(\%)\end{array}$ & $\begin{array}{c}\text { Worse } \\
(\%)\end{array}$ \\
\hline Headache & $87(13 / 15)$ & $13(2 / 15)$ & 0 \\
Sleep disruption & $75(9 / 12)$ & $25(3 / 12)$ & 0 \\
Short-term memory & $92(11 / 12)$ & $8(1 / 12)$ & 0 \\
Cognition & $93(14 / 15)$ & $7(1 / 15)$ & 0 \\
Energy level & $87(13 / 15)$ & $13(2 / 15)$ & 0 \\
Post-traumatic stress & $50(2 / 5) \mathrm{P}$ & $50(3 / 5) \mathrm{P}$ & 0 \\
$\quad$ disorder symptoms (P), & $(2 / 3) \mathrm{N}$ & $(1 / 3) \mathrm{N}$ & \\
$\quad$ or nightmares (N) & & $18(2 / 11)$ & 0 \\
Short temper/irritability & $82(9 / 11)$ & $18(2 / 15)$ & 0 \\
Mood swings & $87(13 / 15)$ & $13(5 / 11)$ & 0 \\
Imbalance & $55(6 / 11)$ & $45(5 / 4)$ & 0 \\
Fine motor & $75(3 / 4)$ & $25(1 / 4)$ & \\
$\quad$ incoordination & & & 0 \\
Decreased hearing & $20(2 / 10)$ & $80(8 / 10)$ & 0 \\
Tinnitus & $37(3 / 8)$ & $63(5 / 8)$ & 0 \\
Depression & $93(13 / 14)$ & $7(1 / 14)$ & 0 \\
Arthralgias & 0 & $100(5 / 5)$ & 0 \\
Photophobia & $44(4 / 9)$ & $44(4 / 9)$ & $11(1 / 9)$ \\
\hline
\end{tabular}

Combined symptoms from subjects' prioritized symptom list and primary author's standard questionnaire.
Table 4. Abnormal Physical Finding Changes (15 Subjects)

\begin{tabular}{|c|c|c|c|}
\hline $\begin{array}{l}\text { Abnormal } \\
\text { physical finding }\end{array}$ & $\begin{array}{l}\text { Better } \\
(\%)\end{array}$ & $\begin{array}{c}\text { No change } \\
(\%)\end{array}$ & $\begin{array}{c}\text { Worse } \\
\%\end{array}$ \\
\hline \multicolumn{4}{|l|}{ Tests of balance: } \\
\hline Tandem gait & $100(14 / 14)$ & 0 & 0 \\
\hline $\begin{array}{l}\text { Romberg (eyes closed, } \\
\text { hands at sides, feet } \\
\text { together } \times 30 \mathrm{sec} \text { ) }\end{array}$ & $93(14 / 15)$ & $7(1 / 15)$ & 0 \\
\hline $\begin{array}{l}\text { Unterberger exam } \\
\text { (arms outstretched, } \\
\text { eyes closed, marching } \\
\text { in place } \times 30 \mathrm{sec} \text { ) }\end{array}$ & $87(7 / 8)$ & $13(1 / 8)$ & 0 \\
\hline \multicolumn{4}{|l|}{ Tests of coordination: } \\
\hline Finger to nose & $91(10 / 11)$ & $9(1 / 11)$ & 0 \\
\hline Heel to shin & $100(5 / 5)$ & 0 & 0 \\
\hline Dysdiadochokinesis & $100(5 / 5)$ & 0 & 0 \\
\hline Rapid finger tapping & $90(9 / 10)$ & $10(1 / 10)$ & 0 \\
\hline \multicolumn{4}{|l|}{ Motor tests: } \\
\hline $\begin{array}{l}\text { Focal weakness: upper } \\
\text { or lower extremity }\end{array}$ & $71(5 / 7)$ & $29(2 / 7)$ & 0 \\
\hline $\begin{array}{l}\text { Deep knee bend: } \\
\text { strength and stability }\end{array}$ & $75(6 / 8)$ & $12.5(1 / 8)$ & $12.5(1 / 8)$ \\
\hline Tremor & $100(5 / 5)$ & 0 & 0 \\
\hline \multicolumn{4}{|l|}{ Sensory tests: } \\
\hline Focal hypesthesia & $89(8 / 9)$ & $11(1 / 9)$ & 0 \\
\hline
\end{tabular}

medication (1/9) increased analgesic medication use. Psychoactive medications pre-HBOT were as follows: selective serotonin reuptake inhibitors/serotonin norepinephrine reuptake inhibitors/aytpical antipsychotics/atypical antidepressants (9 subjects), anxiolytic/hypnotics (8), anticonvulsants (5), anti-migraine (4), narcotics (3), vasodilators (2), muscle relaxants (2), antihistamine/antiemetics (1), cholinesterase inhibitor (1), and stimulants (1). Nine subjects were taking more than one medication, one subject was taking one medication, and five subjects were on no psychoactive medications.

At 6-month phone follow-up 11/12 subjects (92\%) who reported improvement on the majority of the symptoms on their prioritized symptom list after 40 HBOTs had maintained this improvement. One of the three subjects who did not report initial improvement now reported improvement in the majority of symptoms on his prioritized list.

\section{Psychometric testing, affective, TBI/PCS symptom, and quality-of-life measures}

Change from pre- to post-HBOT on the neuropsychological outcome variables is shown in Table 5. Significant improvement was recorded on 7 of 13 measures at $p<0.05$ to $p<0.001$ level and beyond. Global intellectual function and measures of frontal lobe executive function (Working Memory and Stroop Test) showed the largest improvements. The Full Scale IQ increased nearly a full standard deviation, an average of 14.8 points, from 95.8 to $110.6(p<0.001)$. The WMS-IV Working Memory Index increased 9.9 points, from a preHBOT average of 97.0 to a post-HBOT average of 106.9 $(p=0.003)$. The Stroop Color/Word Interference score improved 11.0 points, from a mean of 84.3 to 95.3 after HBOT $(p<0.001)$. 
Table 5. Pre- to Post-HbOt Change for Neuropsychological Outcome Variables

\begin{tabular}{|c|c|c|c|c|}
\hline Outcome variables ${ }^{\mathrm{a}}$ & $\begin{array}{c}\text { Pre-HBOT } \\
\text { mean } \pm S D(15) \\
\text { median (range) }\end{array}$ & $\begin{array}{l}\text { Post-HBOT } \\
\text { mean } \pm \text { SD }(15) \\
\text { median }(\text { range })\end{array}$ & $\begin{array}{l}\text { Pre:post } \\
\text { diff } \pm S D \\
95 \% C I^{\mathrm{b}}\end{array}$ & $\begin{array}{l}\text { Significance } \\
\text { of pre to post }\end{array}$ \\
\hline \multirow[t]{2}{*}{ Full scale $\mathrm{IQ}^{\mathrm{d}}$} & $95.8 \pm 8.4$ & $110.6 \pm 10.3$ & $14.8 \pm 7.4$ & $p<0.001$ \\
\hline & $98(80-106)$ & $110(97-129)$ & CI: 10.7 to 18.9 & \\
\hline \multirow{2}{*}{ Delayed memory (WMS-IV) } & $97.7 \pm 13.3$ & $106.9 \pm 15.4$ & $9.2 \pm 14.3$ & $p=0.026$ \\
\hline & $94(76-125)$ & $107(80-142)$ & CI: 1.3 to 17.1 & \\
\hline \multirow[t]{2}{*}{ Rivermead Paragraph } & $9.5 \pm 2.4(15)$ & $7.5 \pm 3.6(15)$ & $-2.1 \pm 3.7$ & $p=0.049$ \\
\hline & $10(6-14)$ & $8(2-13)$ & $\mathrm{CI}:-4.1$ to -0.0 & \\
\hline \multirow{4}{*}{$\begin{array}{l}\text { Working memory } \\
\text { (WMS-IV) } \\
\text { Stroop color/word interference }\end{array}$} & $97.0 \pm 13.6$ & $106.9 \pm 13.1$ & $9.9 \pm 10.3$ & $p=0.003^{\mathrm{e}}$ \\
\hline & $91(85-131)$ & 105 (88-127) & CI: 4.1 to 15.6 & \\
\hline & $84.3 \pm 12.2$ & $95.3 \pm 12.8$ & $11.0 \pm 9.2$ & $p<0.001$ \\
\hline & $80(65-108)$ & $94(67-118)$ & CI: 6.0 to 16.2 & \\
\hline \multirow[t]{2}{*}{ TOVA $^{f}$ inattention } & $73.3 \pm 29.6(15)$ & $75.8 \pm 27.2(15)$ & $2.5 \pm 22.8$ & $p=0.514$ \\
\hline & $86(40-107)$ & $85(40-107)$ & CI: -10.1 to 15.2 & \\
\hline \multirow[t]{2}{*}{ TOVA impulsivity } & $89.6 \pm 24.9(15)$ & $98.6 \pm 23.1(15)$ & $9 \pm 16.2$ & $p=0.041$ \\
\hline & $90(40-123)$ & $107(40-118)$ & CI: 0.0 to 18.0 & \\
\hline \multirow[t]{2}{*}{ TOVA reaction time } & $93.1 \pm 22.5(15)$ & $99.1 \pm 14.6(15)$ & $5.9 \pm 19.3$ & $p=0.254$ \\
\hline & $99(53-120)$ & $103(70-123)$ & CI: -4.8 to 16.6 & \\
\hline \multirow[t]{2}{*}{ TOVA variability } & $64.4 \pm 28.7$ & $75.3 \pm 24.6$ & $10.9 \pm 20.2$ & $p=0.045$ \\
\hline & $45(40-111)$ & $80(40-111)$ & CI: -0.2 to 22.1 & \\
\hline \multirow[t]{2}{*}{ Finger tap dominant hand } & $90.9 \pm 18.3(15)$ & $98.6 \pm 15.0(15)$ & $7.7 \pm 20.7$ & $p=0.174$ \\
\hline & $93(55-118)$ & $98(75-130)$ & CI: -3.8 to 19.2 & \\
\hline \multirow[t]{2}{*}{ Finger tap non-dominant } & $90.0 \pm 21.5(15)$ & $94.0 \pm 25.2(15)$ & $4 \pm 18.5$ & $p=0.416$ \\
\hline & $95(40-118)$ & $91(40-130)$ & CI: -6.2 to 14.2 & \\
\hline \multirow[t]{2}{*}{ Grooved pegboard dominant } & $88.9 \pm 19.8(15)$ & $96.8 \pm 18.8(15)$ & $7.9 \pm 12.4$ & $p=0.028$ \\
\hline & $88(55-124)$ & $98(65-129)$ & CI: 1.0 to 14.7 & \\
\hline \multirow[t]{2}{*}{ Grooved pegboard non-dominant } & $84.0 \pm 22.0(15)$ & $87.3 \pm 22.8(15)$ & $3.3 \pm 15.3$ & $p=0.423$ \\
\hline & $85(40-120)$ & $85(40-118)$ & CI: -5.2 to 11.8 & \\
\hline
\end{tabular}

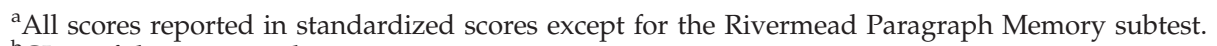

${ }^{\mathrm{b}} \mathrm{CI}$, confidence interval.

${ }^{c} p$ Values are by the paired Student's $t$-test, unless the data were not normally distributed, in which case the non-parametric Wilcoxon signed-ranks test was used.

${ }_{\mathrm{d} P r e-H B O T}$ was Wechsler Adult Intelligence Scale-IV, and Post-HBOT was Wechsler Abbreviated Scale of Intelligence.

enp, non-parametric Wilcoxon signed-ranks test.

${ }^{\mathrm{f}}$ TOVA, Test of Variables of Attention.

IQ, intelligence quotient; WMS-IV, Wechsler Memory Scale Working Memory Index; HBOT, hyperbaric oxygen therapy.

Change in memory was slightly smaller but significant and clinically meaningful on the WMS-IV. The WMS-IV Delayed Memory Index increased 9.2 points, from 97.7 to 106.9 $(p=0.026)$. The Rivermead Paragraph subtest showed a decrease from the pre-HBOT score of 9.5 units recalled to a postHBOT score of 7.5 units recalled $(p=0.049)$.

Improvement in attention was found on several measures but not on all. The TOVA measures of Impulsivity $(p=0.041)$ and Variability $(p=0.045)$ both showed significant increases from pre- to post-HBOT. The TOVA Inattention and Reaction Time measures improved a few points from pre- to postHBOT, but neither was significant.

Only one measure of motor speed and fine-motor coordination (Grooved Pegboard for the dominant hand) showed a significant improvement (7.9 points, $p=0.028)$. Dominant hand motor speed (Finger Tapping Test) increased from a standard score of 90.9 to 98.6 , but failed to reach significance $(p=0.174)$. Neither the Finger Tapping Test nor the Grooved Pegboard pre- to post-HBOT scores were significant for the non-dominant hand.

Table 6 presents the pre- and post-HBOT changes for outcome variables of emotional recovery from PTSD, anxiety, and depression, symptoms of post-concussion, and the subjects' ratings of the percentage of normal they felt for cogni- tive, physical, and emotional functioning. All 8 variables showed a significant improvement from pre- to post-HBOT. On the PTSD Checklist-Military, the average score dropped 20.3 points, from 67.4 to 47.1 ( $p<0.001)$. After HBOT 8 of 14 subjects no longer met the PCL-M threshold criteria for a diagnosis of PTSD. The Rivermead Post Concussion Symptoms Questionnaire average score dropped 15.6 points, from 39.7 pre-HBOT to 24.1 after treatment $(p=0.0002)$. Together, these two measurements indicated a major improvement in the symptoms of PTSD and PCS. Consistent with these findings, the subjects reported a significant drop in depression (PHQ-9; $p<0.001)$ and anxiety (GAD-7; $p=0.007)$, and a concomitant increase in their perceived quality of life $(p=0.003)$. One component of the PHQ-9 addressed suicidal ideation on a four point scale: 0 , none; 1 , several days in last 2 weeks; 2 , more than $1 / 2$ of the days of last 2 weeks; and 3 , nearly every day. The suicidal ideation component of the PHQ-9 improved after treatment by an average of $0.40 \pm 0.63$ points. This improvement was significant by the Wilcoxon test $(p=0.048)$. As a group, the subjects felt that they were less than $50 \%$ back to normal for cognitive, physical, and emotional function when they started treatment. They reported a mean increase of 17.4 points for cognitive function $(p=0.002), 19.5$ points for physical function $(p<0.001)$, and 28.8 points for emotional 
Table 6. Significance of Pre- to Post-HbOT Change for Psychological Outcome Variables

\begin{tabular}{|c|c|c|c|c|}
\hline Outcome variables & $\begin{array}{c}\text { Pre-HBOT } \\
\text { mean } \pm \text { SD (15) } \\
\text { median (range) }\end{array}$ & $\begin{array}{c}\text { Post-HBOT } \\
\text { mean } \pm \text { SD (15) } \\
\text { median (range) }\end{array}$ & $\begin{array}{l}\text { Pre:Post } \\
\text { diff } \pm S D \\
95 \% \mathrm{CI}\end{array}$ & $\begin{array}{l}\text { Significance } \\
\text { of pre to post }\end{array}$ \\
\hline Rivermead PCS & $\begin{array}{l}39.7 \pm 6.0 \\
\quad 40(27-47)\end{array}$ & $\begin{array}{r}24.1 \pm 12.6 \\
26(0-42)\end{array}$ & $\begin{array}{l}-15.6 \pm 12.8 \\
\text { CI: }-22.7 \text { to }-8.5\end{array}$ & $p=0.0002$ \\
\hline PCL-M & $\begin{array}{l}67.4 \pm 10.5 \\
\quad 68(48-84)\end{array}$ & $\begin{array}{l}47.1 \pm 16.0 \\
46(24-69)\end{array}$ & $\begin{array}{l}-20.3 \pm 18.2 \\
\text { CI: }-30.4 \text { to }-10.2\end{array}$ & $p<0.001$ \\
\hline PHQ-9 Depression & $\begin{array}{l}16.6 \pm 4.9 \\
18(5-24)\end{array}$ & $\begin{array}{l}8.2 \pm 4.7 \\
\quad 7(2-17)\end{array}$ & $\begin{array}{l}-8.4 \pm 7.4 \\
\text { CI: }-12.5 \text { to }-4.3\end{array}$ & $p<0.001$ \\
\hline GAD-7 Anxiety & $\begin{array}{l}12.7 \pm 5.8 \\
14(4-21)\end{array}$ & $\begin{array}{l}7.9 \pm 5.3 \\
7(0-21)\end{array}$ & $\begin{array}{l}-4.8 \pm 5.8 \\
\text { CI: }-8.0 \text { to }-1.6\end{array}$ & $p=0.007$ \\
\hline Perceived QOL & $\begin{array}{l}81 \pm 37 \\
\quad 74(29-154)\end{array}$ & $\begin{array}{l}114 \pm 36 \\
125(42-161)\end{array}$ & $\begin{array}{l}33 \pm 36 \\
\quad \text { CI: } 13 \text { to } 53\end{array}$ & $p=0.003$ \\
\hline $\begin{array}{l}\% \text { Back to normal: } \\
\text { Cognitive }\end{array}$ & $\begin{array}{l}49.7 \pm 17.0 \\
\quad 50(20-85)\end{array}$ & $\begin{array}{l}68.9 \pm 20.0 \\
75(30-95)\end{array}$ & $\begin{array}{l}19.2 \pm 17.9 \\
\text { CI: } 9.3 \text { to } 29.1\end{array}$ & $p<0.001$ \\
\hline $\begin{array}{l}\% \text { Back to normal: } \\
\text { Physical }\end{array}$ & $\begin{array}{l}46.7 \pm 22.2 \\
\quad 45(10-85)\end{array}$ & $\begin{array}{l}67.5 \pm 18.5 \\
70(25-90)\end{array}$ & $\begin{array}{l}20.9 \pm 16.3 \\
\text { CI: } 11.8 \text { to } 29.9\end{array}$ & $p<0.001$ \\
\hline $\begin{array}{l}\text { \% Back to normal: } \\
\text { Emotional }\end{array}$ & $\begin{array}{c}32.3 \pm 19.9 \\
30(5-80)\end{array}$ & $\begin{array}{l}63.2 \pm 20.5 \\
\quad 65(30-90)\end{array}$ & $\begin{array}{l}30.9 \pm 21.7 \\
\text { CI: } 18.8 \text { to } 42.9\end{array}$ & $p<0.001$ \\
\hline
\end{tabular}

GAD-7, Generalized Anxiety Disorder 7-item scale; QOL, Quality of Life; PHQ-9, nine-item Patient Health Questionnaire; PCL-M, PTSD Checklist-Military; PCS, Post Concussion Symptoms; HBOT, hyperbaric oxygen therapy; SD, standard deviation; CI, confidence interval.

function $(p<0.001)$, increases of $39 \%, 45 \%$, and $96 \%$, respectively.

\section{SPECT brain blood flow imaging}

SPECT regional cerebral blood flow (rCBF) indices are presented in Table 7: MCP, SD, and CV of counts/pixel in each ROI. MCP, SD, and CV were compared from first scan (pre-HBOT) to after 1 HBOT and after $40 \mathrm{HBOTs}$, and from after 1 HBOT to after 40 HBOTs. Significant changes are shown in Table 8.

SPECT demonstrated significant increases in MCP in the right hemisphere only from baseline to post-1 HBOT $(30,120$, and $150^{\circ}$ gray matter and $120^{\circ}$ white matter ROIs); there were no significant changes from baseline to post- 40 HBOTs. In the left hemisphere SPECT demonstrated significant increases in MCP from baseline to post- 1 HBOT $\left(30,60,120\right.$, and $150^{\circ}$ gray matter ROIs and 60 and $120^{\circ}$ white matter ROIs), and from baseline to post- 40 HBOTs $\left(120^{\circ}\right.$ gray matter and 60 and $120^{\circ}$ white matter ROIs).

SPECT demonstrated significant decreases in the SD of counts/pixel in the right hemisphere only from baseline to post- 1 HBOT $\left(90\right.$ and $150^{\circ}$ gray matter ROIs and $60^{\circ}$ white matter ROI); there were no significant changes from baseline to post-40 HBOTs. However, there were significant increases (a reversal of effect) from post- 1 to post-40 HBOTs (60 and $150^{\circ}$ gray matter ROIs). In the left hemisphere SPECT demonstrated significant decreases in the SD of counts/pixel only

Table 7. MCP, SD, and CV Counts/Pixel SPECT Brain Blood Flow in Right (R) and Left (L) Hemisphere ROIs of a Single Transverse Slice in the Centrum Semiovale

\begin{tabular}{|c|c|c|c|c|c|c|c|c|c|}
\hline ROI & $\begin{array}{c}\text { MCP } \\
\text { Pre }\end{array}$ & $\begin{array}{c}M C P \\
P 1\end{array}$ & $\begin{array}{c}M C P \\
P 40\end{array}$ & $\begin{array}{l}\text { SD } \\
\text { Pre }\end{array}$ & $\begin{array}{l}S D \\
P 1\end{array}$ & $\begin{array}{l}S D \\
P 40\end{array}$ & $\begin{array}{l}\text { CV } \\
\text { Pre }\end{array}$ & $\begin{array}{l}C V \\
P 1\end{array}$ & $\begin{array}{l}C V \\
P 40\end{array}$ \\
\hline $30^{\circ} \mathrm{G}$ & 63 & 4 & 9 & 9 & 8 & 0 & 1 & 74 & $4.28 \pm 1.95$ \\
\hline $0 c$ & & & & & & & & & \\
\hline & \pm 263 & $1500 \pm 305$ & & & & & & & \\
\hline${ }^{\circ} \mathrm{G}$ & $16 \pm 248$ & $1604 \pm 294$ & $1605 \pm 311$ & & & & & & $3.57 \pm 1.06$ \\
\hline & $1501 \pm 253$ & $1740 \pm 329$ & $1767 \pm 348$ & $71.0 \pm 34.0$ & & & $6 \pm 1.72$ & & 2.13 \\
\hline $60^{\circ} \mathrm{YW}$ & $20 \pm 156$ & $899 \pm 171$ & $983 \pm 235$ & .7 & 4 & & 61 & & \\
\hline $\mathrm{R} 120^{\circ} \mathrm{W}$ & & & & $.7 \pm 2$ & & & & & \\
\hline & $1405 \pm 260$ & $1593 \pm 305$ & 1600 & & & & & $3.60 \pm$ & \\
\hline & $1367 \pm 274$ & $1558 \pm$ & $1614 \pm 3$ & $85.0 \pm$ & $68.8 \pm 2$ & $79.3=$ & $6.40=$ & $4.41 \pm$ & 5.23 \\
\hline & $338 \pm$ & & & 6 & $521+$ & .5 & $4.72=$ & $3.37=$ & 3.62 \\
\hline & $5 \pm 246$ & 1 & & 0 & 4 & & & 2. & .77 \\
\hline & $36 \pm 250$ & $1728 \pm 3$ & $1747 \pm 3$ & & $45.9 \pm$ & & & 270 & 4.04 \\
\hline & $818 \pm 120$ & $998 \pm 292$ & $1013 \pm 254$ & $56.7 \pm 26.1$ & $56.0 \pm 50.0$ & & & $5.13 \pm 2.59$ & $6.91 \pm 2.82$ \\
\hline $\mathrm{L} 120^{\circ} \mathrm{W}$ & $745 \pm 117$ & $882 \pm 136$ & $922 \pm 248$ & $63.4 \pm 19.1$ & $53.8 \pm 18.6$ & $56.8 \pm 29.9$ & $8.58 \pm 2.53$ & $6.15 \pm 2.01$ & $6.20 \pm 3.10$ \\
\hline
\end{tabular}

$30,60,90,120$, and $150^{\circ}$ gray $(\mathrm{G})$ matter and 60 and $120^{\circ}$ white $(\mathrm{W})$ matter, before HBOT (Pre), post- 1 HBOT (P1), and post-40 HBOTs (P40).

$\mathrm{MCP}$, mean number of counts/pixel; SD, standard deviation of counts/pixel; CV, coefficient of variation; HBOT, hyperbaric oxygen therapy. 
Table 8. Significant Changes in MCP, SD, and CV Counts/Pixel

\begin{tabular}{lccccccccc}
\hline Measurement & MCP PP1 & MCP PP40 & MCP P1,40 & SD PP1 & SD PP40 & SD P1,40 & CV PP1 & CV PP40 & CV P1,40 \\
\hline R-30-G & 0.038 & 0.170 & 0.987 & 0.471 & 0.389 & 0.174 & 0.098 & 0.772 & 0.152 \\
R-60-G & 0.052 & 0.120 & 0.861 & 0.441 & 0.056 & 0.012 & 0.012 & 0.204 \\
R-90-G & 0.076 & 0.217 & 0.967 & 0.020 & 0.282 & 0.053 & $<0.001$ & 0.865 \\
R-120-G & 0.031 & 0.134 & 0.991 & 0.616 & 0.895 & 0.539 & 0.155 & 0.604 & 0.018 \\
R-150-G & 0.015 & 0.051 & 0.825 & 0.035 & 0.302 & 0.017 & 0.008 & 0.879 & 0.044 \\
R-60-W & 0.068 & 0.055 & 0.244 & 0.011 & 0.080 & 0.715 & 0.002 & 0.007 \\
R-120-W & 0.045 & 0.261 & 0.804 & 0.057 & 0.476 & 0.510 & 0.011 & 0.107 & 0.964 \\
L-30-G & 0.028 & 0.098 & 0.936 & 0.001 & 0.388 & 0.003 & $<0.001$ & 0.821 & 0.004 \\
L-60-G & 0.025 & 0.059 & 0.630 & 0.210 & 0.582 & 0.192 & 0.048 & 0.127 & 0.679 \\
L-90-G & 0.069 & 0.066 & 0.563 & 0.185 & 0.417 & 0.557 & 0.009 & 0.068 \\
L-120-G & 0.025 & 0.040 & 0.756 & 0.052 & 0.842 & 0.151 & 0.012 & 0.240 & 0.463 \\
L-150-G & 0.041 & 0.075 & 0.874 & $<0.001$ & 0.937 & 0.003 & $<0.001$ & 0.180 \\
L-60-W & 0.014 & 0.034 & 0.884 & 0.599 & 0.050 & 0.083 & 0.055 & 0.989 \\
L-120-W & 0.014 & 0.003 & 0.568 & 0.237 & 0.545 & 0.714 & 0.029 & 0.037 \\
\hline
\end{tabular}

Changes shown are from pre-HBOT to after the first HBOT (PP1; post-1 HBOT minus pre), pre-HBOT to after 40 HBOTs (PP40; post-40 HBOTs minus pre), and post-first HBOT to post-40 HBOTs (P1,40; post-40 HBOTs minus post- 1 HBOT), in the right (R) and left (L) hemisphere ROIs at $30,60,90,120$, and $150^{\circ}$ of gray (G) matter, and 60 and $120^{\circ}$ of white (W) matter of a transverse SPECT slice in the centrum semiovale.

Positive changes were assigned to significant increases in $\mathrm{MCP}$, and decreases in SD and CV are shaded blue. Near positive significant changes in MCP, SD, and CV are shaded green. Negative changes were assigned to decreases in $\mathrm{MCP}$, and increases in SD and CV and are shaded red. Numerical figures are $p$ values. Note differences in the right and left hemisphere MCPs for post-first and post-40th HBOT significant reductions in the SD and CV after the first HBOT in both gray and white matter, and in the white matter only after 40 HBOTs, while a reversal of this effect, significant increases, were seen in the SD and CV between the first and 40th HBOT in mostly gray matter sites and one white matter site.

$\mathrm{MCP}$, mean number of counts/pixel; SD, standard deviation of counts/pixel; CV, coefficient of variation; HBOT, hyperbaric oxygen therapy.

from baseline to post- 1 HBOT ( 30 and $150^{\circ}$ gray matter ROIs). There were significant increases (reversal of effect) from baseline to post- 40 HBOTs $\left(60^{\circ}\right.$ white matter ROI) and post- 1 to post-40 HBOTs (30 and $150^{\circ}$ gray matter ROIs).

SPECT demonstrated significant reductions in the CV of counts/pixel in the right hemisphere from baseline to post-1 $\operatorname{HBOT}\left(60,90\right.$, and $150^{\circ}$ gray matter and 60 and $120^{\circ}$ white matter ROIs), and from baseline to post- 40 HBOTs $\left(60^{\circ}\right.$ white matter ROI). There were significant increases (reversal of effect) from post- 1 HBOT to post- 40 HBOTs $\left(60,90\right.$, and $150^{\circ}$ gray matter ROIs). In the left hemisphere SPECT demonstrated significant reductions in the CV of counts/pixel from baseline to post- 1 HBOT $\left(30,60,90,120\right.$, and $150^{\circ}$ gray matter ROIs and $120^{\circ}$ white matter ROI), and from baseline to post40 HBOTs $\left(120^{\circ}\right.$ white matter ROI). However, there were significant increases (reversal of effect) from post- 1 HBOT to post- 40 HBOTs ( 30 and $150^{\circ}$ gray matter ROIs and $60^{\circ}$ white matter ROI).

\section{SPM results}

Initial statistical parametric maps (SPM) with significance set at $p<0.01$ with family-wise error correction (FWE) for multiple comparisons showed diffuse improvements in brain blood flow. In order to separate clusters into discrete anatomical locations the significance level was raised to $p<0.001$ with FWE. This analysis revealed that 85 clusters had significantly improved after 1 HBOT. The 11 most significant regions of change occurred in the precentral, temporal, thalamic, and occipital regions, and are displayed in Table 9 and Figure 2 (the eleventh was included because of its location in the motor area). There were no significant differences when comparing the second (after 1 HBOT) and the third (after 40
HBOTs) scans at this level of significance. However, when comparing the third scan to the baseline scan the significance level threshold had to be raised to $p<0.0001$ with FWE to achieve cluster separation into discrete anatomical areas. At this level of significance 50 significant clusters were identified

Table 9. Top 11 Clusters of Voxels Showing Significant INCREASES IN BRAin BloOd Flow AFTER 1 HBOT COMPAREd to BASELINe Scans

Increases in rCBF post-first HBOT

\begin{tabular}{|c|c|c|c|c|c|}
\hline \multirow[b]{2}{*}{ Brain area } & \multirow{2}{*}{$\begin{array}{l}\text { Cluster } \\
\text { size } K_{E}\end{array}$} & \multirow[b]{2}{*}{$T$} & \multicolumn{3}{|c|}{ Coordinates } \\
\hline & & & $X$ & $Y$ & Z \\
\hline 1. Precentral left & 946 & 30.18 & -32 & -22 & 56 \\
\hline 2. Temporal lobe left & 615 & 29.64 & -62 & -26 & 10 \\
\hline 3. Precuneus right & 1663 & 27.81 & 22 & -52 & 24 \\
\hline 4. Thalamus right & 89 & 25.15 & 10 & -6 & 8 \\
\hline 5. Post-central right & 211 & 24.44 & 42 & -30 & 66 \\
\hline 6. Occipital left & 427 & 23.36 & -20 & -72 & 20 \\
\hline 7. Lingual left & 50 & 23.22 & -20 & -68 & -10 \\
\hline $\begin{array}{l}\text { 8. Temporal inferior } \\
\text { to mid-lateral right }\end{array}$ & 103 & 22.66 & 44 & -2 & -36 \\
\hline $\begin{array}{l}\text { 9. Temporal } \\
\text { mid-lateral left }\end{array}$ & 266 & 22.33 & -52 & -64 & -4 \\
\hline $\begin{array}{l}\text { 10. Frontal inferior } \\
\text { triangle left }\end{array}$ & 75 & 22.24 & -48 & 26 & 8 \\
\hline $\begin{array}{l}\text { 11. Superior motor } \\
\text { area right }\end{array}$ & 134 & 22.09 & 12 & 16 & 50 \\
\hline
\end{tabular}

Significance level raised to $p<0.001$ with family-wise error correction (FWE).

HBOT, hyperbaric oxygen therapy; rCBF, regional cerebral blood flow. 


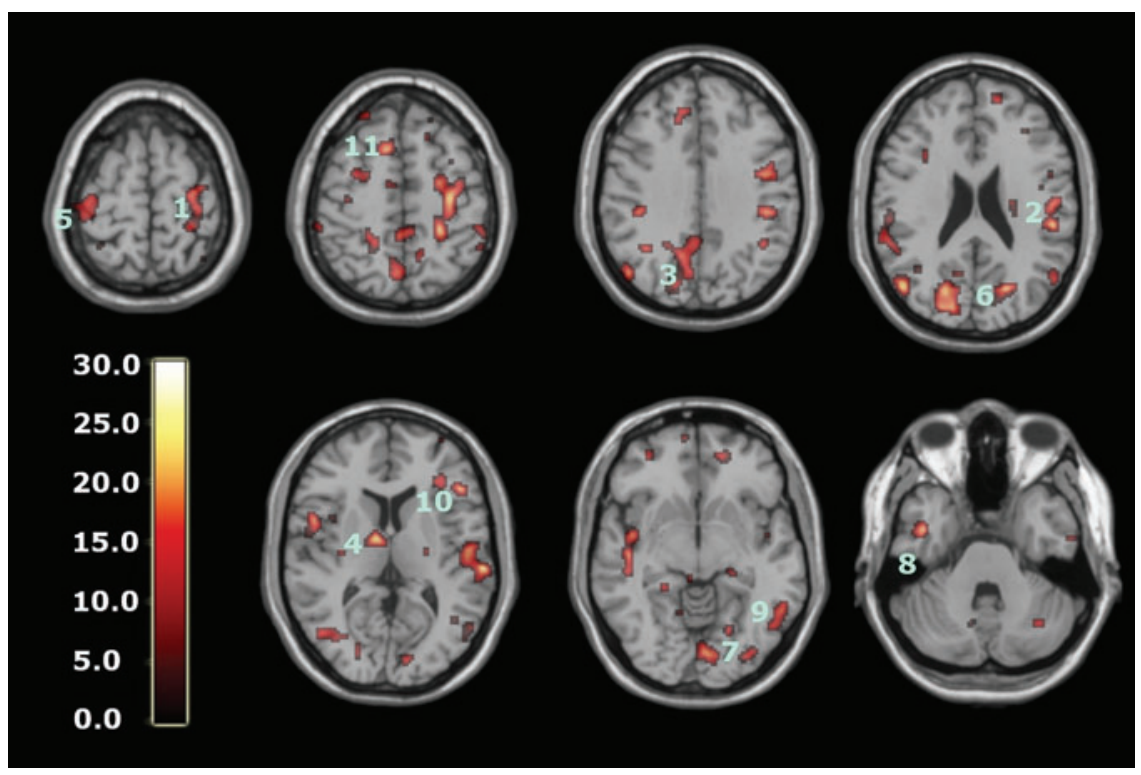

FIG. 2. Fusion of significant single photon emission computed tomography (SPECT) clusters after 1 HBOT with standard reference MRI T1 transverse image. Numbers correspond to the top 11 significant clusters at the $p<0.001$ level labeled in Table 9, numerically in order from highest $\mathrm{T}$ value to lowest. Significant clusters incidentally occurring on the same slices are also depicted. (Color bar shows relative amplitude of rCBF improvement; rCBF, regional cerebral blood flow; HBOT, hyperbaric oxygen therapy; MRI, magnetic resonance imaging).

(Table 10 and Figure 3). The most significant change was in the right frontal region after 40 HBOTs.

To compare significant increases in brain blood flow after 1 HBOT to changes after 40 HBOTs, a significance level of $p<0.001$ was chosen. Cortical maps of these analyses dem-

Table 10. Top 10 Clusters of Voxels Showing Significant Increases in Brain Blood Flow AFTER 40 HBOTs COMPAREd to BAseline SCANS

Increases in rCBF post-fortieth HBOT

\begin{tabular}{|c|c|c|c|c|c|}
\hline \multirow[b]{2}{*}{ Brain area } & \multirow{2}{*}{$\begin{array}{l}\text { Cluster } \\
\text { size } K_{E}\end{array}$} & \multirow[b]{2}{*}{$T$} & \multicolumn{3}{|c|}{ Coordinates } \\
\hline & & & $X$ & $Y$ & Z \\
\hline $\begin{array}{l}\text { 1. Frontal mid } \\
\text { to mid orbital right }\end{array}$ & 314 & 39.94 & 38 & 42 & \\
\hline $\begin{array}{l}\text { 2. Occipital superior } \\
\text { to calcarine right }\end{array}$ & 7126 & 38.78 & 22 & -78 & \\
\hline $\begin{array}{l}\text { 3. Temporal pole superior } \\
\text { to insula left }\end{array}$ & 112 & 34.14 & -34 & 6 & -1 \\
\hline $\begin{array}{l}\text { 4. Temporal Superior } \\
\text { to post-central left }\end{array}$ & 438 & 33.8 & -55 & -12 & \\
\hline $\begin{array}{l}\text { 5. Cerebellum to } \\
\text { temporal inferior left }\end{array}$ & 1146 & 33.47 & -22 & -76 & - \\
\hline 6. Calcarine left & 212 & 29.99 & -6 & 96 & \\
\hline $\begin{array}{l}\text { 7. Frontal inferior triangle } \\
\text { to mid-lateral orbital left }\end{array}$ & 370 & 27.79 & -36 & 42 & \\
\hline $\begin{array}{l}\text { 8. Parietal superior } \\
\text { to inferior right }\end{array}$ & 401 & 27.11 & 34 & -52 & \\
\hline $\begin{array}{l}\text { 9. Precuneus to para } \\
\text { central lobule right }\end{array}$ & 693 & 26.89 & 10 & -44 & \\
\hline 10. Superior motor area left & 228 & 26 & -2 & 18 & 5 \\
\hline
\end{tabular}

Significance level raised to $p<0.0001$ with family-wise error correction (FWE).

HBOT, hyperbaric oxygen therapy; $\mathrm{rCBF}$, regional cerebral blood flow. onstrate more widespread significant increases in brain blood flow after 40 HBOTs (Fig. 4).

To illustrate the overlap of brain areas with increased brain blood flow after 1 HBOT that also showed increased brain blood flow after 40 HBOTs, the analysis after 40 HBOTs $(p<0.001)$ was repeated using the clusters affected by 1 HBOT $(p<0.01)$ as a mask. Seventy-five significant clusters were discovered, with the top 10 most significant shown in Table 11 and Figure 5.

A separate analysis tested the hypothesis that $\mathrm{rCBF}$ in the hippocampus should improve after HBOT given symptomatic and measured WMS memory improvements. The changes after 1 HBOT were compared to the changes after 40 HBOTs (Fig. 6). After 1 HBOT significant changes $(p<0.001)$ were seen in hippocampal regions on both sides of the brain. The most significant changes were seen in a cluster in the inferior lateral left hippocampus $\left(\mathrm{t}=17 ; \mathrm{K}_{\mathrm{E}}=93\right.$; coordinates $-28,-8$, and -24$)$, followed by a cluster in the superior medial left hippocampus $\left(\mathrm{t}=14.86, \mathrm{~K}_{\mathrm{E}}=139\right.$; coordinates $-22,-28$, and -6$)$. The largest cluster was seen in the right medial hippocampus ( $t=12.69$; coordinates $24,-22$, and -16). After 40 HBOTs the significant changes in the hippocampus remained on both sides of the brain $(p<0.001)$. The most significant changes in hippocampal rCBF were seen in the lateral right hippocampus $\left(t=23.95 ; K_{E}=626\right.$; coordinates $42,-18$, and -18$)$, followed by the left medial hippocampus $\left(\mathrm{t}=14.81 ; \mathrm{K}_{\mathrm{E}}=366\right.$, coordinates $-20,-20$, and -16).

\section{Discussion}

\section{Safety of the HBOT protocol}

In this preliminary report of the effect of 40 HBOTs on blast-induced chronic mild to moderate PCS and PTSD we observed that HBOT 1.5 ATA is safe with no major side 


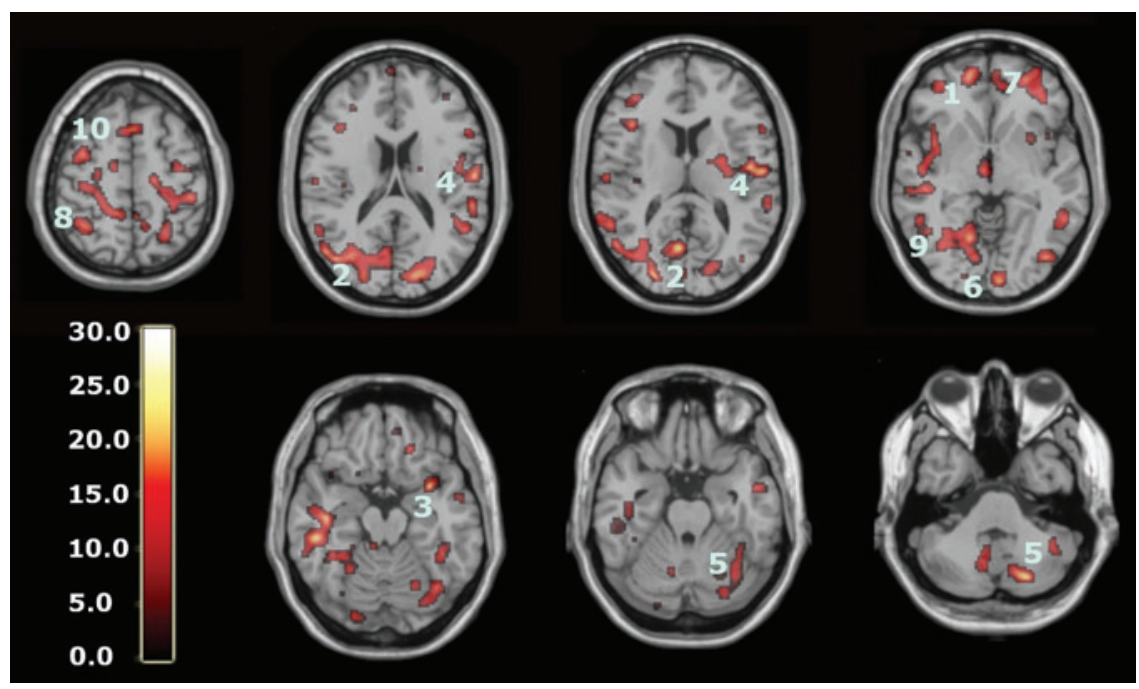

FIG. 3. Fusion of significant single photon emission computed tomography (SPECT) clusters after 40 HBOTs with standard reference MRI T1 transverse image. Numbers correspond to the top 10 significant clusters at the $p<0.0001$ level labeled in Table 10 numerically in order from highest $\mathrm{T}$ value to lowest. Significant clusters incidentally occurring on the same slices are also depicted. The color bar shows relative amplitude of $\mathrm{rCBF}$ improvement rCBF, regional cerebral blood flow; HBOT, hyperbaric oxygen therapy; MRI, magnetic resonance imaging).

effects or complications. Although the number of subjects is small, this lack of major side effects is consistent with ours and others' previous experience with similar low-pressure HBOT in patients with more severe chronic TBI (Golden et al., 2002; Harch et al., 1994,1996a; Neubauer et al., 1994; Harch and Neubauer, 1999,2004a,2009b,2009c), but differs from a report by Lin and associates (Lin et al., 2008) on HBOT in moderate to severe TBI, where they found that $9 \%$ of the patients experienced seizures. The dosage of HBOT in the Lin study was 2.0 ATA for $1.5 \mathrm{~h}$ at depth for 20 treatments, compared to our 1.5 ATA for $60 \mathrm{~min}$ total treatment time. The Lin seizure rate is 300 times the seizure frequency in the general HBOT population at 2.4-2.5 ATA (Clark, 2009), and 30 times the seizure rate at 2.45 ATA in acutely carbon monoxide-poisoned patients (Hampson et al., 1996). The greater seizure frequency in the Lin study is likely due to the combination of more severe brain injury, earlier treatment, no air breaks during HBOT, and the dose of 2.0 ATA for $1.5 \mathrm{~h}$. Seizures at 1.5 ATA have only been reported with prolonged series of treatment, and much greater numbers of HBOTs (Harch, 2002), than those employed in the present study.

Reversible MEBT occurred in 5 of 16 subjects. Most of these occurred during the prodromal and early clinical phase of acute URIs. URI is an uncommonly recorded adverse event in HBOT, but twice/day dosing is also atypical for chronic hyperbaric indications. It is not our preferred dosing schedule, but was chosen due to limitations of time, resources, finances, and out-of-state location in this subject population. The mild immunosuppression of HBOT (Rossignol, 2007) and twice per day dosing may have contributed to the $25 \%$ URI rate.

Four of the subjects (25\%) experienced a transient deterioration in symptomatology at approximately 20 HBOTs. This has not been reported previously in hyperbaric medicine. We speculate that this mid-point in the protocol represents a transition in brain wound adaptation/ transformation to the repetitive effects of intermittent hyperoxia. Due to the self-limited course of this deterioration and the final response to the full course of treatment we

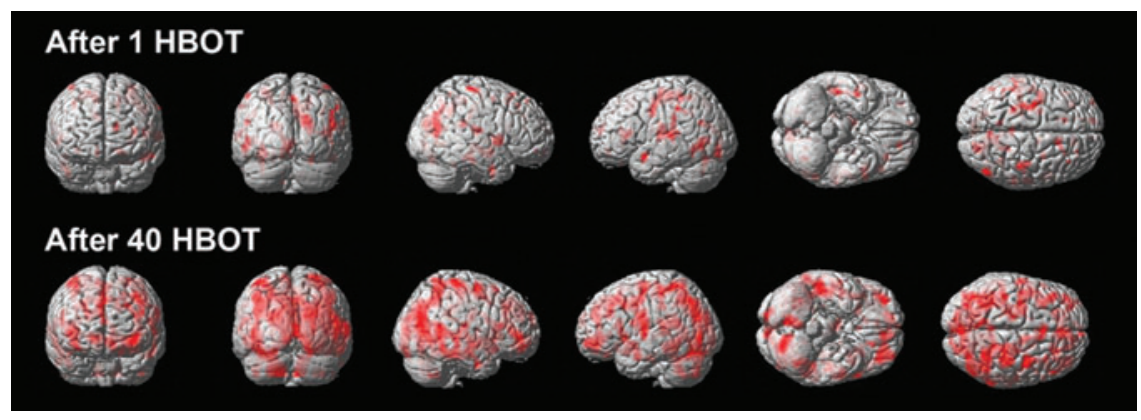

FIG. 4. Cortical views from the front, back, right, left, inferior, and superior aspects show effects of 1 HBOT (top row) and 40 HBOTs (bottom row) at a significance level of $p<0.001$. Significant increases are shown in red (HBOT, hyperbaric oxygen therapy). 
Table 11. Top 10 Clusters of Voxels Showing Significant Increases in Brain Blood Flow Common TO BRAIN SCANS AFTER 1 HBOT $(p<0.01$ WITH FWE) AND 40 HBOTs $(P<0.001$ WITH FWE $)$

Increases in rCBF post-40 HBOTs masked by scan post-1 HBOT

\begin{tabular}{lrrrrrr}
\hline & & & \multicolumn{3}{c}{ Coordinates } \\
\cline { 5 - 7 } Brain area & $\begin{array}{c}\text { Cluster } \\
\text { size } K_{E}\end{array}$ & $T$ & $X$ & $Y$ & $Z$ \\
\hline 1. Occipital superior & 1581 & 38.78 & 22 & -78 & 28 \\
$\quad$ to temporal right & & & & & \\
2. Temporal superior left & 514 & 33.8 & -56 & -12 & 4 \\
3. Temporal right & 360 & 31.84 & 52 & -32 & -20 \\
4. Precentral left & 917 & 29.4 & -44 & -2 & 40 \\
5. Parietal superior right & 11 & 26.2 & 34 & -52 & 60 \\
6. Cuneus to occipital left & 405 & 24.04 & -12 & -86 & 16 \\
7. Cerebellum left & 71 & 22.88 & -38 & -58 & 20 \\
8. Cerebellum & 24 & 22.02 & 14 & -56 & 10 \\
$\quad$ to lingual right & & & & & \\
9. Post-central to & supra-marginal right & 203 & 21.51 & 36 & -32 & 68 \\
10. Rolandic operculum right & 85 & 21.11 & 60 & -18 & 12 \\
\hline
\end{tabular}

FWE, family-wise error correction; HBOT, hyperbaric oxygen therapy; rCBF, regional cerebral blood flow.

conclude that there is no justification for cessation of HBOT during this transition.

\section{Effectiveness of HBOT for blast TBI and PTSD}

The remarkable findings in this study were the significant improvements in self-reported symptoms, physical exam changes, PCS symptoms, perceived quality of life questionnaires, affective measures (general anxiety, depression, suicidal ideation, and PTSD), cognitive measures (memory, working memory, attention, and FSIQ score), and SPECT brain blood flow imaging. The magnitude of improvement was consistent across all domains measured. These findings were mirrored by a reciprocal reduction or elimination of psychoactive and narcotic prescription medication usage in $64 \%$ of those subjects for whom they were prescribed. Spontaneous improvement as an explanation for all of these findings is inconsistent with the natural history of PCS and PTSD 2.8 years after injury.
Reduction in headaches and increase in FSIQ/cognitive function evidenced effectiveness of HBOT 1.5 ATA in the treatment of blast TBI/PCS cerebral wounds. Headache is a marker of blast-induced PCS and distinguishes PCS from PTSD (Hoge et al., 2008). In our study 13/15 (87\%) patients reported a substantial reduction in headaches during the 30 days they received HBOT. A reduction in headache and improvement in PCS symptoms (39\% reduction in RPCSQ, $p=0.0002)$ is consistent with the treatment of the extracerebral marker of PCS, as well as the associated underlying biological injury caused by TBI. This biological wound is established in our subjects due to their loss of consciousness (Lidvall, 1975; Symonds, 1962).

FSIQ increased 14.8 points to $110.6(p<0.001)$. As a global measure of cognitive function this increase is consistent with the patients' self-reported $40 \%$ cognitive improvement, the global nature of blast brain injury, and the global improvement in blood flow seen on SPECT. Some of the IQ increase could be explained by WASI FSIQ overestimation (Axelrod, 2002) compared to the WAIS-III, but the WASI has been validated in other adult heterogeneous clinical samples (Ryan, 2003; Hays, 2002). Our study was performed on a relatively homogenous patient group. The consistency of our findings despite different ways of measuring (WASI and PBNRS) argues against a significant contribution from a WASI flaw, and is consistent with the conclusion that the HBOT did improve overall cognitive functioning.

Memory and frontal lobe function (simple sustained attention, working memory, and more complex attention) improved from what would appear to be "average" or "normal" levels to what the subjects considered to be more their "normal" levels. Our results are very similar to cognitive improvements in a controlled chronic severe TBI HBOT study (Golden et al., 2006) and case report (Hardy, 2007). While only $26 \%$ of the subjects were TBI patients in the Golden study, 35 HBOTs in 35 days caused a significant 7.19-point increase in Stroop Color/Word score compared to normal and chronic brain injury controls, both of whom had similar 30- to 35-day test/retest intervals. The test/retest effect across 1- and 2week intervals is 3.83 points (Franzen et al., 1987). The combined effect of Golden and test/retest $(7.19+3.83=11.02)$ is nearly identical to the 11.0 point seen increase in our study.

Changes in motor speed and fine motor coordination reached significance on only one of four measures, the

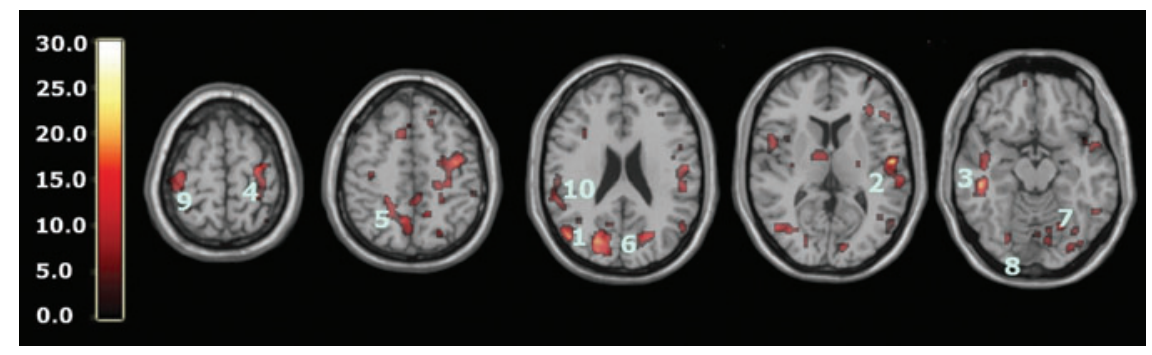

FIG. 5. Fusion of significant single photon emission computed tomography (SPECT) clusters after 40 HBOTs masked by clusters after 1 HBOT with standard reference MRI T1 transverse image. Numbers correspond to the top 10 significant clusters in Table 11 at the $p<0.001$ level when masked inclusively by results after 1 scan at the $p<0.01$ level, numerically ordered from highest to lowest $\mathrm{T}$ value. Significant clusters incidentally occurring on the same slices are also depicted. The color bar shows relative amplitude of rCBF improvement (rCBF, regional cerebral blood flow; HBOT, hyperbaric oxygen therapy; MRI, magnetic resonance imaging). 


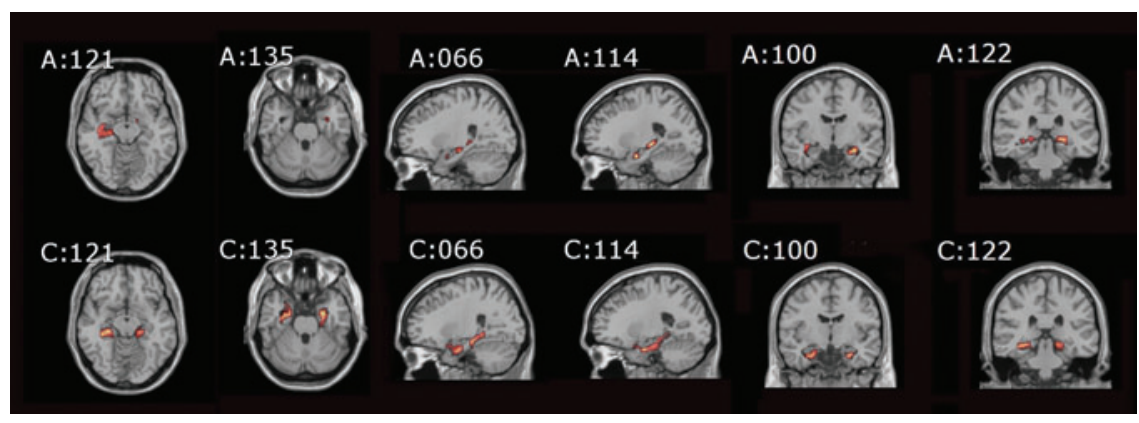

FIG. 6. Fusion of significant single photon emission computed tomography (SPECT) hippocampal increases in rCBF with standard reference MRI T1 transverse, sagittal, and coronal slices after 1 HBOT (row A) and 40 HBOTs (row C; $p<0.001$ with FWE; FWE, family-wise error correction; rCBF, regional cerebral blood flow; HBOT, hyperbaric oxygen therapy; MRI, magnetic resonance imaging.

Grooved Pegboard for the dominant hand, while the P.I. recorded improvements of coordination in $90-100 \%$ of subjects who had abnormalities on baseline testing. Possible explanations for this discrepancy include: (1) testing of different sizes and groups of muscles (finger/hand for the psychometric tests versus the entire upper and lower extremities on physical exam); (2) investigator bias/non-blinding; (3) qualitative (physical exam) versus quantitative (psychometric) testing; (4) small number in the study.

The Rivermead Behavioral Memory (RBM) Paragraph Delayed Recall was the sole significant negative cognitive outcome. The RBM is only one subtest of a larger test, and was added because the test offered alternative forms of the paragraph for retesting purposes. The negative result may be a function of the limited range of the test, unequal difficulty of the different paragraphs, small number, problems with sustained attention immediately after our intensive HBOT schedule, or a true negative effect of HBOT on this component of memory.

The SPECT findings were as impressive as the cognitive improvements, and were consistent with the bi-hemispheric increases in SPECT regional cortical blood flow reported by Neubauer and Golden (Golden et al., 2002). Both texture and SPM analyses showed consistent and significant improvements in blood flow after 1 and 40 HBOTs compared to baseline, no significant difference in blood flow between 1 and 40 HBOTs, yet considerable overlap of the areas with improved blood flow after 1 and 40 HBOTs. SPM also revealed more widespread significant increases in blood flow after 40 versus 1 HBOT (more voxels and brain regions) compared to baseline, and compared to texture analysis which showed the opposite, fewer ROIs with significant increases in blood flow after 40 versus 1 HBOTs. This discrepancy was due to an increased variance in blood flow after 40 HBOTs versus 1 HBOT that is evident on the reversal of SD and CV improvements in primarily gray matter ROIs from 1 to 40 HBOTs (2/3 right and left hemisphere white matter ROIs maintained the improvement in SD and CV after 40 HBOTs that were seen after 1 HBOT). Some of the increased variance might be explained by the timing of imaging (within $4 \mathrm{~h}$ after the first HBOT and $48 \mathrm{~h}$ after the 40th HBOT), and the intensive twice/day, 5 days/week HBOT schedule. This increased variance is not captured on SPM due to the different analytical and statistical methods.

Significant improvements in SPECT occurred after both 1 and 40 HBOTs; however, by historical precedent and design symptoms, cognition, and QoL were only tested after 40 HBOTs. The symptomatic, cognitive, and QoL improvements evolved over the course of the treatment and no subject claimed significant symptomatic improvement after the first HBOT session. The dichotomous findings of SPECT improvement after 1 and 40 HBOTs and neurological function only after 40 HBOTs, and the differential effect of 40 HBOTs on white versus gray matter SPECT texture analysis strongly suggest different physiological effects of 1 and 40 HBOTs on the injured brain at different points in the treatment process. Furthermore, the differential effect of 40 HBOTs on white versus gray matter is consistent with a biological effect of repetitive HBOT 1.5 ATA on the primary injury site in mild to moderate TBI, the white matter (Kraus et al., 2007; Lipton et al., 2009).

An unexpected finding was the confirmation of a reduction in PTSD that was symptomatically observed in our first published case of PCS/PTSD (Harch et al., 2009a). In the present study subjects achieved a $30 \%$ reduction in PTSD scores in a 30-day period. A biological substrate for this HBOT effect is difficult to identify. Symptomatically, combat blastinduced PCS is inextricably interwoven with blast-induced PTSD. PCS and PTSD share some common biological pathways, processes, and anatomy in the brain (Kennedy et al., 2007). The hippocampus, in particular, is a pathological target in both PCS (Umile, 2002) and PTSD (Bremner, 2007; Wang, 2010; Woon and Hedges, 2008). HBOT treatment of hippocampal PCS injury may explain some of the observed effect on PTSD symptom reduction seen in our study.

Explanatory mechanisms for the HBOT effects are numerous. Neubauer and associates (Neubauer et al., 1990) demonstrated that increased brain blood flow after a single HBOT in chronic cerebral ischemia (the Neubauer effect) predicted subsequent neurological improvement with repetitive HBOT. Ischemia is a known pathological process in TBI (Gaetz, 2004). Focal ischemia causes a post-transcriptional metabolic/protein synthesis impairment to neurons, termed the ischemic freeze (Hossman, 1993). The first HBOT may override this ischemic freeze, consistent with Siddiqui's demonstration of improved oxygen capacitance of non-CNS ischemic tissue (Siddiqui et al., 1997). The increase in blood flow on SPECT after 1 HBOT session in our study may reflect this reversal of impaired protein synthesis. Simultaneously, it may test vascular reserve capacity similarly to the Wada test (Vorstrup, 1988). 
The global improvements in brain blood flow after 1 HBOT in our subjects were associated with improved function after 40 HBOTs, thus supporting the Neubauer effect's prediction of neurological improvement. SPM analysis demonstrated considerable overlap of the areas with improved blood flow after 1 HBOT with those after 40 HBOTs, indicating that the areas identified on SPECT by the Neubauer effect are likely those responsible for neurological improvement after 40 HBOTs. We have demonstrated the Neubauer effect in severe chronic TBI patients (Harch and Neubauer, 1999, 2004a,2009b,2009c; Harch et al., 1994,1996a; Neubauer et al., 1994), along with a pattern shift on SPECT after the first HBOT. The pattern shift consists of normalization (a relative decrease in high and increase in low blood flow; Harch and Neubauer, 1999,2004b; Harch, et al., 1996a) that is captured by a reduction in SD and CV in this study. The first HBOT would not be expected to improve function, however, likely due to the limited impact of a single HBOT on blast-induced degenerated white matter (Bauman et al., 2009).

The increased blood flow on SPECT, variance in MCP change, and improved neurological function seen after 40 HBOT sessions suggests a set of mechanisms different from those that occur after 1 HBOT session. We propose that these mechanisms are the typical trophic mechanisms of HBOT in chronic non-central nervous system wounds (Gesell, 2009). Repetitive HBOT stimulates angiogenesis in chronic non-CNS wounded tissue (Marx et al., 1990), most likely by genomic effects (Godman et al., 2009), and has been shown to increase blood vessel density in injured hippocampus in our chronic rat TBI model, where the progenitor of this HBOT protocol was tested (Harch et al., 2007). HBOT-induced increased hippocampal blood vessel density in this model highly correlated with improved spatial learning and memory. In our subjects SPECT SPM analysis showed significant improvements in blood flow in the hippocampus, while our subjects achieved significant gains in memory. These blood flow and memory improvements seen in our subjects are consistent with a trophic effect of HBOT on chronic brain wounding in the hippocampus, and possible healing/reinnervation of denervated tissue (Bauman et al., 2009).

Other mechanisms may contribute to the HBOT effects seen in our study. A single hyperbaric oxygen reoxygenation session causes prolonged excitability and neural plasticity of hippocampal neurons after exposure to hypoxia (Garcia et al., 2010), consistent with the Neubauer effect generated in this study. Repetitive HBOT has shown increased neurogenesis and cerebral blood flow in chronic global ischemia (Zhang et al., 2010). Zhang and associates administered repetitive HBOT 30 days after ischemic insult, similar to the 50-day delay in our animal model (Harch et al., 2007). Neurogenesis has been shown to occur in association with angiogenesis (Palmer et al., 2000). As mentioned above, angiogenesis is a known trophic mechanism of HBOT, and may be responsible for the increased blood vessel density seen in our animal model (Harch et al., 2007). HBOT has also been shown to cause the release of bone marrow stem cells into the peripheral circulation (Thom et al., 2006). Peripheral stem cells are known to cross the blood-brain barrier (Mezey et al., 2003).

The limitations of the present study were a lack of confirmation of post-injury brain MRI results in some subjects, unblinded investigators (except for the SPECT brain imaging SPM analysis), and lack of a control group. The lack of con- firmation of brain MRI findings in a few subjects could confound study results only by inadvertent inclusion of nonclinically-apparent neurological disease that was manifest on MRI alone. We believe this is a very remote possibility; these young men were highly fit pre-military, underwent regular fitness evaluations while in the military, and had no premorbid disqualifying conditions. All symptomatology commenced with the incident blast and was present continuously since the blast. Routine late MRI evaluations in mild to moderate TBI are usually negative, consistent with the majority of the scans in our subjects. We presume the few missing data points would similarly be normal or non-contributory.

Investigator bias and placebo effects possibly contributed to the magnitude of some of the effects we measured, but are unlikely to account for the majority of the effects or the consistency and magnitude of the effects seen across all domains, particularly SPECT. Investigator bias could be present in the P.I.'s symptom and physical exam recording, and in S.R.A.'s neuropsychological testing, but it does not explain the significant SPECT findings for which separate independent analyses, one of which was blinded, were performed by E.F.F. in North Dakota and D.A. and D.V.T. in California. None of the SPECT co-investigators interacted with the subjects, and they performed their analyses months after the subjects had completed their final imaging. Importantly, the blinded SPECT analyst, D.V.T., produced the most significant statistical results.

Placebo effects cannot be entirely ruled out; however, there are multiple arguments against this notion. Treatment effect size in two meta-analyses of randomized placebo-controlled trials versus observational studies performed on the same treatments has been shown to be very similar (Benson and Hartz, 2000; Concato et al., 2000). This suggests that placebo effects are overestimated in observational studies such as ours. Placebo effects on many of the cognitive measures in our study have been reported to be smaller than the changes we found with HBOT for FSIQ and WMS Visual Immediate and Delayed Memory (Doraiswamy et al., 2007), for Stroop Reaction Time (Calabrese et al., 2008), and for Stroop Color/ Word raw score (Jorge et al., 2010). The placebo effects reported on SPECT in psychiatric disease, in healthy individuals, and in neurological disease have shown focal changes in regional cerebral blood flow (Beauregard, 2009), most commonly in the inferior frontal gyrus, striatum, and rostral anterior cingulate cortex (Jarcho et al., 2009). The global diffuse changes we measured have not been reported. In addition, it is highly improbable that a placebo effect could account for the multiplicity of differential changes on SPECT seen after 1 and 40 HBOTs using two different forms of mathematical/ statistical analyses. Lastly, the parallel improvements in memory scores and hippocampal blood flow are inconsistent with a placebo effect.

Test/retest practice effects could explain some of the cognitive improvements; however, practice effects do not fully explain our measured increases for seven reasons. (1) Practice effects on the WAIS-III FSIQ over a mean 34.6-day retest interval have been shown to be $2.0-3.2$ points across all age groups, 6 points in the 16- to 29-year-old group, and decrease with age; our subjects averaged 30 years old (Tulsky and Zhu, 1997). They have also been shown to increase 6 points over $3-$ or 6-month retest times (Basso et al., 2002). Six points is $41 \%$ of the measured FSIQ increase on the WAIS-IV in our subjects. 
(2) The bulk of practice effects occur on the first retest (Bartels et al., 2010; Falleti et al., 2006), and our subjects had been cognitively tested at least once before our pre-HBOT testing session. Second and third retest (third and fourth tests) effects should have been smaller than 6 points. (3) Working memory has been shown to be among the most resistant to practice/ retest effects (Bartels et al., 2010; Basso et al., 2002). Our subjects averaged a 9.9-point statistically significant improvement. (4) Practice effects are usually studied in normal individuals with intact memory function. Intact memory is a prerequisite for learning/practice effects. In individuals with impaired memory function, such as our subjects, practice effects may be less (Basso et al., 2002). (5) We used the alternate form WASI for the post-treatment IQ test in order to minimize practice effects. (6) A Stroop Color/Word score increase in a controlled HBOT study of chronic brain injury produced results similar to ours (Golden et al., 2006). (7) Stroop Color/ Word test/retest effects across 1- and 2-week intervals are 3.83 points (Franzen et al., 1987), and our increase was 11.0 points.

Our results were achieved with half (40 HBOTs) of our normal protocol (80 HBOTs) on an accelerated twice/day schedule due to time and fiscal constraints. Through clinical experience, clinical research, and an animal pilot study that compared sham HBOT, 40, and 80 HBOTs (Harch et al., 1996b), we found greater cognitive and blood flow improvements (in an animal model; Harch et al., 2007), and clinical and blood flow improvements (in human cases) with 80 HBOTs, but the cases were primarily chronic moderate to severe TBI (vide supra). Neubauer and Golden (Golden et al., 2002) reported progressively greater blood flow in a case series of chronic severe brain-injured patients receiving 70 low pressure HBOTs. Recently, Wright and colleagues (2010) reported the effectiveness of our HBOT 1.5 ATA protocol in two airmen with blast-induced PCS, using 40 and 80 HBOTs (for persistent symptoms after 40 HBOTs). Our subjects finished HBOT with partial improvement in their symptoms. It is likely that additional HBOT sessions would be beneficial.

In conclusion, application of a lower-pressure protocol of 40 HBOTs at 1.5 ATA to a 16-subject cohort of military subjects with blast-induced chronic PCS and PTSD was found to be safe. One fourth of the subjects experienced transient clinical deterioration halfway through the protocol and one subject did not finish. Simultaneously, as a group the 15 subjects experienced notable improvements in symptoms, abnormal physical exam findings, cognitive testing, PCS and PTSD symptom questionnaires, quality-oflife questionnaires, depression and anxiety indices, and SPECT brain blood flow imaging that are inconsistent with the natural history of PCS 2.8 years post-injury. The symptomatic improvements were present at 6-month phone follow-up in $92 \%$ of subjects who reported improvement after 40 HBOTs. More objective psychometric testing and SPECT imaging were not performed to confirm the durability of the HBOT treatment effect. Sixty-four percent of the patients on psychoactive and narcotic prescription medications were able to decrease or eliminate use of these medications. These data are preliminary and need confirmation with larger numbers of subjects or with a stronger design such as a randomized or Bayesian study.

\section{Acknowledgments}

The authors thank The Marine Corps Law Enforcement Foundation, The Semper Fi Fund, The Coalition to Salute Americas Heroes, the Harch Hyperbaric Research Fund of the Baromedical Research Institute of New Orleans, Mr. Caleb Gates, New Orleans Natural Resource Group, Rubie and Bryan Bell, Martin and Margaret Hoffmann, John and Virginia Weinmann, Dr. Warren Thomas, Joan C. White, Health Freedom Foundation, Soldiers Angels, Operation Homefront Louisiana, The Audubon Society, Mr. Theodore Solomon, New Orleans Steamboat Company, the National WWII Museum, and Westwego Swamp Boat Tours for their generous donations. We thank Mr. Martin Hoffmann, ex-Secretary of the Army (President Gerald Ford) for his indefatigable fundraising efforts, Sean Bal and Ray Crowell, our hyperbaric technicians for their expert and safe delivery of hyperbaric oxygen therapy, Wanda Phillips for review of all of the study records, and Amy Trosclair of the BRI for overseeing the handling and disbursement of funds.

\section{Author Disclosure Statement}

Dr. Harch owns a small consulting company called Harch Hyperbarics, Inc., which has no contracts. For Dr. Andrews no competing financial interests exist. Juliette Lucarini, R.N. is a tenant in common ownership of Harch Hyperbarics, Inc. For Claire Aubrey, Dr. Fogarty, and Dr. Staab no competing financial interests exist. Dr. Pezzullo is an independent statistical consultant for whom no competing financial interests exist. For Dr. Amen and Derek Taylor no competing financial interests exist. Dr. Van Meter has a hyperbaric equipment leasing company and contracts with hospitals to provide hyperbaric medicine physician staffing.

\section{References}

Andrykowski, M.A., Cordova, M.J., Studts, J.L., and Miller, T.W. (1998). Posttraumatic stress disorder after treatment for breast cancer: Prevalence of diagnosis and use of the PTSD ChecklistCivilian Version (PCL-C) as a screening instrument. J. Consult. Clin. Psychol. 66, 586-590.

Ashburner, J., and Friston, K.J. (1999). Nonlinear spatial normalization using basis functions. Hum. Brain Mapp. 7, 254-266.

Axelrod, B.N. (2002). Validity of the Wechsler abbreviated scale of intelligence and other very short forms of estimating intellectual functioning. Assessment 9, 17-23.

Bartels, C., Wegrzyn, M., Ackermann, V., and Ehrenreich, H. (2010). Practice effects in healthy adults: a longitudinal study on frequent repetitive cognitive testing. BMC Neurosci. 16, 111-118.

Basso, M.R., Carona, F.D., Lowery, N., and Axelrod, B.N. (2002). Practice effects on the WAIS-III across 3 and 6 month intervals. Clin. Neuropsychologist 16, 57-63.

Bauman, R.A., Ling, G., Tong, L., Januszkiewicz, A., Agoston, D., Delanerolle, N., Kim, Y., Ritzel, D., Bell, R., Ecklund, J., Armonda, R., Bandak, F., and Parks, S. (2009). An introductory characterization of a combat-casualty-care relevant swine model of closed head injury resulting from exposure to explosive blast. J. Neurotruama 26, 841-860.

Beauregard, M. (2009). Effect of mind on brain activity: evidence from neuroimaging studies of psychotherapy and placebo effect. Nord. J. Psychiatry 63, 5-16. 
Benson, K., and Hartz, A.J. (2000). A comparison of observational studies and randomized, controlled trials. N. Engl. J. Med. 342, 1878-1886.

Bremner, J.D. (2007). Neuroimaging in posttraumatic stress disorder and other stress-related disorders. Neuroimaging Clin. N. Am. 17, 523-538.

Calabrese, C., Gregory, W.L., Leo, M., Kraemer, D., Bone, K., and Oken, B. (2008). Effects of a standardized Bacopa monnieri extract on cognitive performance, anxiety, and depression in the elderly: a randomized, double-blind, placebo-controlled trial. J. Altern. Complement. Med. 14, 707-713.

Carlson, C.F., Kehle, S.M., Meis, L.A., Greer, N., MacDonald, R., Rutks, I., Sayer, N.A., Dobscha, S.K., and Wilt, T.J. (2010). Prevalence, assessment, and treatment of mild traumatic brain injury and posttraumatic stress disorder: A systematic review of the evidence. J. Head Trauma Rehabil. Jul 13 [Epub ahead of print].

Centers for Medicare and Medicaid Services. National Coverage Determination (NCD) Hyperbaric Oxygen Therapy (20.29). National Coverage Determinations (NCD) Manual, Publication Number 100-3, Chapter 1, Part 1, Section 20.29, effective date 6/19/2006. http://www.cms.hhs.gov/mcd/viewncd.asp? ncd_id $=20.29 \&$ ncd_version $=3 \&$ basket $=$ ncd $\% 3 \mathrm{~A} 20.29 \% 3 \mathrm{~A} 3 \% 3 \mathrm{~A}$ Hyperbaric + Oxygen + Therapy

Clark, J.M. (1993). Oxygen toxicity, in: Chapter 6, The Physiology and Medicine of Diving, 4th ed. P. Bennett and D. Elliott (eds). W.B. Saunders: London, pps. 121-169.

Clark, J. (2009). Side effects, in: Hyperbaric Oxygen Therapy Indications, 12th ed. The Hyperbaric Oxygen Therapy Committee Report. L.B. Gesell (ed). Undersea and Hyperbaric Medical Society: Durham, NC, pps. 217-218.

Concato, J., Shah, N., and Horwitz, R.I. (2000). Randomized, controlled trials, observational studies, and the hierarchy of research designs. N. Engl. J. Med. 342, 1887-1892.

Cyceron, Plateforme d'Imagerie Biomedicale. http://www .cyceron.fr/web/aal_anatomical_automatic_labeling.html

Doraiswamy, P.M., Babyak, M.A., Hennig, T., Trivedi, R., White, W.D., Mathew, J.P., Newman, M.F., and Blumenthal, J.A. (2007). Donepezil for cognitive decline following coronary artery bypass surgery: a pilot randomized controlled trial. Psychopharmacol. Bull. 40, 54-62.

Dougherty, G. (1996). Quantitative CT in the measurement of bone quantity and bone quality for assessing osteoporosis. Med. Eng. Phys. 18, 557-568.

Falleti, M.G., Maruff, P., Collie, A., and Darby, D.G. (2006). Practice effects associated with the repeated assessment of cognitive function using the CogState Battery at 10-minute, one week and one month test-retest intervals. J. Clin. Exp Neurop. 28, 1095-1112.

Franzen, M.D., Tishelman, A.C., Sharp, B.H., and Friedman, A.G. (1987). An investigation of the test-retest reliability of the Stroop Color-Word Test across two intervals. Arch. Clin. Neuropsych. 2, 265-272.

Gaetz, M. (2004). The neurophysiology of brain injury. Clin. Neurophys. 115, 4-18.

Garcia, A.J., Putnam, R.W., and Dean, J.B. (2010). Hyperbaric hyperoxia and normobaric reoxygenation increase excitability and activate oxygen-induced potentiation in CA1 hippocampal neurons. J. Appl. Physiol. 109, 804-819.

Gavin, D.R., Ross, H.E., and Skinner, H.A. (1989). Diagnostic validity of the Drug Abuse Screening Test in the assessment of DSM-III drug disorders. Br. J. Addiction 84, 301-307.

Gesell, L.B. (2009). Chair and Editor, Hyperbaric Oxygen Therapy Indications, 12th ed. The Hyperbaric Oxygen Therapy Com- mittee Report. Durham, NC: Undersea and Hyperbaric Medical Society.

Godman, C.A., Chheda, K.P., Hightower, L.E., Perdrizet, G., Shin, D.-G., and Giardina, C. (2009). Hyperbaric oxygen induces a cytoprotective and angiogenic response in human microvascular endothelial cells. Cell Stress and Chaperones December, DOI 10.1007/s12192-009-0159-0.

Golden, Z., Golden, C.J., and Neubauer, R.A. (2006). Improving neuropsychological function after chronic brain injury with hyperbaric oxygen. Disabil. Rehabil. 28, 1379-1386.

Golden, Z.L., Neubauer, R., Golden, C.J., Greene, L., Marsh, J., and Mleko, A. (2002). Improvement in cerebral metabolism in chronic brain injury after hyperbaric oxygen therapy. Int. J. Neurosci. 112, 119-131.

Greenberg, L. (1996). Test of Variables of Attention Continuous Performance Test. Universal Attention Disorders, Inc.: Los Alamitos, CA.

Hampson, N.B., Simonson, S.G., Kramer, C.C., and Piantadosi, C.A. (1996). Central nervous system oxygen toxicity during hyperbaric treatment of patients with carbon monoxide poisoning. Undersea Hyperb. Med. 23, 215-219.

Harch, P.G., and Neubauer, R.A. (1999). Hyperbaric Oxygen Therapy in Global Cerebral Ischemia/Anoxia and Coma, in: Chapter 18, Textbook of Hyperbaric Medicine, 3rd revised ed. K.K. Jain (ed.). Hogrefe and Huber Publishers: Seattle, pps. 319-345.

Harch, P.G., and Neubauer, R.A. (2004a). Hyperbaric oxygen therapy in global cerebral ischemia/anoxia and coma, in: Chapter 18, Textbook of Hyperbaric Medicine, 4th revised ed. K.K. Jain (ed). Hogrefe and Huber Publishers: Seattle, pps. 223-261.

Harch, P.G., and Neubauer, R.A. (2009b). Hyperbaric oxygen therapy in global cerebral ischemia/anoxia and coma, in: Chapter 19, Textbook of Hyperbaric Medicine, 5th revised ed. K.K. Jain (ed). Hogrefe and Huber Publishers: Seattle, pps. 235-274.

Harch, P.G., Fogarty, E.F., Staab, P.K., and Van Meter, K. (2009a). Low pressure hyperbaric oxygen therapy and SPECT brain imaging in the treatment of blast-induced chronic traumatic brain injury (post-concussion syndrome) and post traumatic stress disorder: a case report. Cases Journal 2, 6538. http://casesjournal.com/casesjournal/article/view/6538

Harch, P.G., Kriedt, C.L., Weisand, M.P., Van Meter, K.W., and Sutherland, R.J. (1996b). Low pressure hyperbaric oxygen therapy induces cerebrovascular changes and improves complex learning/memory in a rat open head bonk chronic brain contusion model. Undersea Hyperb. Med. 23 (Suppl.), 48.

Harch, P.G., Kriedt, C., Van Meter, K.W., and Sutherland, R.J. (2007). Hyperbaric oxygen therapy improves spatial learning and memory in a rat model of chronic traumatic brain injury. Brain Res. 1174, 120-129.

Harch, P.G., Neubauer, R.A., Uszler, J.M., and James, P.B. (2009c). Appendix: Diagnostic Imaging and HBO Therapy, in: Chapter 44, Textbook of Hyperbaric Medicine, 5th revised ed. K.K. Jain (ed). Hogrefe and Huber Publishers: Seattle, pps. 505-519.

Harch, P.G., Neubauer, R.A., Uszler, J.M., and James, P.B. (2004b). Appendix: Diagnostic Imaging and HBO Therapy, in: Chapter 41, Textbook of Hyperbaric Medicine, 4th revised ed. K.K. Jain (ed). Hogrefe and Huber Publishers: Seattle, pps. 471-485.

Harch, P.G. (2002). The dosage of hyperbaric oxygen in chronic brain injury, in: The Proceedings of the 2nd International Symposium on Hyperbaric Oxygenation for Cerebral Palsy 
and the Brain-Injured Child. J.T. Joiner (ed). Best Publishing Co.: Flagstaff, pps. 31-56.

Harch, P.G., Van Meter, K.W., Gottlieb, S.F., and Staab, P. (1994). HMPAO SPECT brain imaging and low pressure HBOT in the diagnosis and treatment of chronic traumatic, ischemic, hypoxic, and anoxic encephalopathies. Undersea Hyperb. Med. 21 (Suppl.), 30.

Harch, P.G., Van Meter, K.W., Neubauer, R.A., and Gottlieb, S.F. (1996a). Use of HMPAO SPECT for assessment of response to $\mathrm{HBO}$ in ischemic/hypoxic encephalopathies, in: Appendix, Textbook of Hyperbaric Medicine, 2nd ed. K.K. Jain (ed). Hogrefe and Huber Publishers: Seattle, pps. 480-491.

Hardy, P., Johnston, K.M., De Beaumont, L., Montgomery, D.L., Lecomte, J.M., Soucy, J.P., Bourbonnais, D., and Lassonde, M. (2007). Pilot case study of the therapeutic potential of hyperbaric oxygen therapy on chronic brain injury. J. Neurol. Sci. 253, 94-105.

Hays, J.R., Reas, D.L., Shaw, J.B. (2002). Concurrent validity of the Wechsler abbreviated scale of intelligence and the Kaufman brief intelligence test among psychiatric inpatients. Psychol. Rep. 90(2), 355-9.

Hoge, C.W., McGurk, D., Thomas, J.L., Cox, A.L., Engel, C.C., and Castro, C.A. (2008). Mild traumatic brain injury in U.S soldiers returning from Iraq. N. Engl. J. Med. 358, 453-463.

Hossman, K.-A. (1993). Disturbances of cerebral protein synthesis and ischemic cell death, in: Volume 96, Progress in Brain Research. K. Kogure, K.-A. Hossman, and B.K. Siesjo (eds). Elsevier Science Publishers: Amsterdam, pps. 161-177.

Jarcho, J.M., Mayer, E.A., and London, E.D. (2009). Neuroimaging placebo effects: new tools generate new questions. Clin. Pharmacol. Ther. 86, 352-354.

Jorge, R.E., Acion, L., Moser, D., Adams, H.P., and Robinson, R.G. (2010). Escitalopram and enhancement of cognitive recovery following stroke. Arch. Gen. Psychiatry 67, 187-196.

Keane, T., Fairbank, J., Caddell, J., Zimering, R., Taylor, K., and Mora, C. (1989). Clinical evaluation of a measure to assess combat exposure. Psychological Assessment 1, 53-55.

Kennedy, J.E., Jaffee, M.S., Leskin, G.A., Stokes, J.W., Leal, F.O., and Fitzpatrick, P.J. (2007). Posttraumatic stress disorder and posttraumatic stress disorder-like symptoms and mild traumatic brain injury. J. Rehabil. Res. Dev. 44, 895-920.

King, N., Crawford, S., Wenden, F., Moss, N., and Wade, D. (1995). The Rivermead Post Concussion Symptoms Questionnaire: a measure of symptoms commonly experienced after head injury and its reliability. J. Neurology 242, 587-592.

Kraus, M.F., Susmaras, T., Caughlin, B.P., Walker, C.J., Sweeney, J.A., and Little, D.M. (2007). White matter integrity and cognition in chronic traumatic brain injury: a diffusion tensor imaging study. Brain 130, 2508-2519.

Kroenke, K., Spitzer, R.L., and Williams, J.B.L. (2001). The PHQ9, validity of a brief depression severity measure. J. Gen. Intern Med. 16, 606-613.

Lesak, M., Howieson, D., and Loring, D. (2004). Neuropsychological Assessment, 4th ed. Oxford University Press: New York, pps. 365-367, 776.

Lidvall, H.F. (1975). Recovery after minor head injury. Lancet 1, 100.

Lin, J.W., Tsai, J.T., Lee, L.M., Lin, C.M., Hung, C.C., Hung, K.S., Chen, W.Y., Wei, L., Ko, C.P., Su, Y.K., and Chiu, W.T. (2008). Effect of hyperbaric oxygen on patients with traumatic brain injury. Acta Neurochir. Suppl. 101, 145-149.

Lipton, M.L., Gulko, E., Zimmerman, M.E., Friedman, B.W., Kim, M., Gellella, E., Gold, T., Shifteh, K., Ardekani, B.A., and Branch, C.A. (2009). Diffusion-tensor imaging implicates pre- frontal axonal injury in executive function impairment following very mild traumatic brain injury. Radiology 252, 816824.

Marx, R.E., Ehler, W.J., Tayapongsak, P., and Pierce, L.W. (1990). Relationship of oxygen dose to angiogenesis induction in irradiated tissue. Am. J. Surg. 260, 519-524.

MAST Revised. (2009). http://counsellingresource.com/quizzes/ alcoholmast/index.html

Mezey, E., Key, S., Vogelsang, G., Szalayova, I., Lange, G.D., and Crain, B. (2003). Transplanted bone marrow generates new neurons in human brains. PNAS 100, 1364-1369.

Neubauer, R.A., Gottlieb, S.F., and Kagan, R.L. (1990). Enhancing "idling" neurons. Lancet, 335, 542.

Neubauer, R.A., Gottlieb, S.F., and Pevsner, N.H. (1994). Hyperbaric oxygen for treatment of closed head injury. South. Med. J. 87, 933-936.

Palmer, T.D., Willhoite, A.R., and Gage, F.H. (2000). Vascular niche for adult hippocampal neurogenesis. J. Comp. Neurol. 425, 479-494.

Patrick, D.L., Danis, M., Southerland, L.I., and Hong, G. (1988). Quality of life following intensive care. J. Gen. Intern Med. 3, 218-223.

Powell, J.M., Machamer, J.E., Temkin, N.R., and Dikmen S.S. (2001). Self-report of extent of recovery and barriers to recovery after traumatic brain injury: a longitudinal study. Arch. Phys. Med. Rehabil. 82, 1025-1030.

PTSD Checklist-Military. (2009). http://wwwnmcphc.med.navy .mil/deployment_health/frm_ptsdmilitary.aspx

Rappaport, M., Hall, K.M., Hopkins, K., Belleza, T., and Cope, D.N. (1982). Disability rating scale for severe head trauma: coma to community. Arch. Phys. Med. Rehabil. 63, 118-123.

Reitan, R.M., and Wolfson, D. (1993). The Halstead-Reitan Neuropsychological Test Battery: Theory and Clinical Interpretation. Neuropsychology Press: Tucson.

Rossignol, D.A. (2007). Hyperbaric oxygen therapy might improve certain patho-physiological findings in autism. Med. Hypotheses 68, 1208-1227.

Ryan, J.J., Carruthers, C.A., Miller, L.J., Souheaver, G.T., Gontkovsky, S.T., Zehr, M.D. (2003). Exploratory factor analysis of the Wechsler Abbreviated Scale of Intelligence (WASI) in adult standardization and clinical samples. Appl. Neuropsychol. 10(4), 252-6.

Siddiqui, A., Davidson, J.D., and Mustoe, T.A. (1997). Ischemic tissue oxygen capacitance after hyperbaric oxygen therapy: a new physiologic concept. Plast. Reconstr. Surg. 99, 148155.

Spitzer, R.L., Kroenke, K., Williams, J.B.W., and Löwe, B. (2006). A brief measure for assessing generalized anxiety disorder: the GAD-7. Arch. Intern Med. 166, 1092-1097.

Sterr, A., Herron, K.A., Hayward, C., and Montaldi, D. (2006). Are mild head injuries as mild as we think? Neurobehavioral concomitants of chronic post-concussion syndrome. BMC Neurol. 6, doi:10.1186/1471-2377-6-7.

Symonds, C. (1962). Concussion and its sequelae. Lancet 1, 1-5. Tanielian, T., and Jaycox, L.H., eds. (2008). Invisible Wounds of War: Psychological and Cognitive Injuries, Their Consequences, and Services to Assist Recovery. Center for Military Health Policy Research, The Rand Corporation: Arlington, VA.

Thom, S.R., Bhopale, V.M., Velazquez, O.C., Goldstein, L.J., Thom, L.H., and Buerk, D.G. (2006). Stem cell mobilization by hyperbaric oxygen. Am. J. Physiol. Heart Circ. Physiol. 290, H1378-H1386.

Tulsky, D., and Zhu, J. (1997). WAIS-III/WMS-III Technical Manual. Psychological Corporation: San Antonio, TX. 
Umile, E.M., Sandel, M.E., Alavi, A., Terry, C.M., and Plotkin, R.C. (2002). Dynamic imaging in mild traumatic brain injury: support for the theory of medial temporal vulnerability. Arch. Phys. Med. Rehabil. 83, 1506-1513.

U.S. Navy Diving Manual, Revision 6, Vol. 1, Sec. 3-9.2.2.2. Symptoms of CNS oxygen toxicity, pps. 42-43. Chapter 3: Underwater Physiology and Diving Disorders. 3-9 Indirect Effects of Pressure on the Human Body. SS521-AG-PRO-010, 9010-LP-106-0957. Published by direction of Commander, Naval Sea Systems Command, 4/15/2008.

Vorstrup, S. (1988). Tomographic cerebral blood flow measurements in patients with ischemic cerebrovascular disease and evaluation of the vasodilatory capacity by the acetazolamide test. Acta Neurol. Scand. 114 (Suppl.), 1-48.

Wang, Z., Neylan, T.C., Mueller, S.G., Lenoci, M., Truran, D., Marmar, C.R., Weiner, M.W., and Schuff, N. (2010). Magnetic resonance imaging of hippocampal subfields in posttraumatic stress disorder. Arch. Gen. Psychiatry 67, 296-303.

WAIS-IV. (2009). http://pearsonassess.com/HAIWEB/Cultures/ en-us/Product Detail.htm?Pid=015-8980-808\&Mode=summary.

WASI. (2009). http://pearsonassess.com/haiweb/cultures/enus / productdetail. $\mathrm{htm}$ ?pid $=015-8981-502$

Wechsler, D. (2001). Wechsler Test of Adult Reading (WTAR)Manual. The Psychological Corporation: San Antonio, TX.

Wilson, B., Cockburn, J., and Baddeley, A. (1985). The Rivermead Behavioural Memory Test. Thames Valley Test Company: Reading, England.
WMS-IV. (2009). http://pearsonassess.com/HAIWEB/Cultures/ en-us / Productdetail.htm?Pid $=8895-800$

Woon, F.L., and Hedges, D.W. (2008). Hippocampal and amygdala volumes in children and adults with childhood maltreatment-related posttraumatic stress disorder: a metaanalysis. Hippocampus 18, 729-736.

Wright, J.K., Zant, E., Groom, K., Schlegel, R.E., and Gilliland, K. (2009). Case report: Treatment of mild traumatic brain injury with hyperbaric oxygen. Undersea Hyperb. Med. 36, 391-399.

Zhang, T., Yang, Q.-W., Wang, S.-N., Wang, J.-Z., Want, Q., Want, Y., and Luo, Y.-J. (2010). Hyperbaric oxygen therapy improves neurogenesis and brain blood supply in piriform cortex in rats with vascular dementia. Brain Inj. 24, 13501357.

Address correspondence to: Paul G. Harch, M.D. Hyperbaric Medicine Department Department of Medicine Section of Emergency and Hyperbaric Medicine Louisiana State University Health Sciences Center 1542 Tulane Avenue, Room 452, Box T4M2 New Orleans, LA 70112

E-mail: paulharchmd@gmail.com

APPENDix

\section{Standardized questionnaire:}

1. Energy level on 1-10 scale (10 was pre-LOC energy level, 0 is inability to get out of bed).

2. Weight change since injury.

3. Mood swings.

4. Irritability/short temper.

5. Mood, 1-10 scale (10 is happiest in life, 0 is not wanting to live).

6. Cranial and cranial nerve symptoms: headache, dizziness, visual symptoms, loss of hearing, tinnitus, vertigo, change in smell/taste, trouble talking, enunciating, swallowing, or chewing.

7. Sensory symptoms: numbness, tingling.

8. Motor: focal or generalized weakness.

9. Incoordination: fine motor (hands/fingers), gross motor (tripping, stumbling, imbalance).

10. Cognitive: trouble thinking/grasping ideas, organizing thoughts, decreased speed of thinking, confusion, problems following directions/instructions, difficulty expressing thoughts/word-finding, forgetfulness, misplacing/ losing things, problems remembering old information or new information, losing one's place in thought or conversation or while driving, going blank, staring episodes, feeling suddenly lost or disoriented, concentration/attention problems, difficulty writing, family or friends commenting on change in personality or behavior.

11. Joint pain or swelling.

12. Incontinence of bowel or bladder.

Neurological exam:

1. Cranial nerves: II-XII.

2. Deep tendon reflexes upper and lower extremities.

3. Motor: tone, mass, tremor, deep knee bend, strength, tiptoe, and heel walking.

4. Sensory: pinprick and touch in the four extremities.

5. Gait: normal, tandem (slow and fast).

6. Pathological reflexes: glabellar, snout, palmomental, grasp, suck, root, Hoffman, Babinski, clonus.

7. Cerebellar: Romberg, finger tapping speed/rhythm, elbow flexion check response, finger-to-nose testing, heel-to-shin gliding, rapid alternating hand movementspalm/dorsal hand thigh slapping. 